\title{
Identification of the active compounds and pharmacological mechanisms of Xiaoqinglong decoction for asthma based on network pharmacology and molecular docking technology
}

Haili Li

Guangxi Medical University https://orcid.org/0000-0003-0728-6147

Jianpeng Zhou

Guangxi Medical University First Affiliated Hospital

\section{Zuyou Wei}

Guangxi Medical University First Affiliated Hospital

\section{Feng Chen}

Ruikang Hospital Affiliated to Guangxi University of Chinese Medicine Jingmin Deng (D ldyy666@163.com )

The First Affiliated Hospital of Guangxi Medical University https://orcid.org/0000-0002-9421-2279

\section{Research}

Keywords: Xiaoqinglong decoction, asthma, Traditional Chinese medicine, network pharmacology, molecular docking

Posted Date: November 24th, 2020

DOI: https://doi.org/10.21203/rs.3.rs-112111/v1

License: (c) (i) This work is licensed under a Creative Commons Attribution 4.0 International License. Read Full License 


\section{Abstract}

Background and purpose

Asthma has become the most common chronic respiratory disease in the world. Xiaoqinglong decoction (XQLD) is described as a commonly used drug for treatment and prevention of asthma for thousands of years, however, its underlying molecular mechanisms have not been clarified completely. Therefore, a network pharmacology and molecular docking technology were used to uncover the active compounds and pharmacological mechanism of XQLD on asthma.

Methods

Bioactive ingredients and targets of XQLD, asthma-related targets were obtained from public databases. Cytoscape software was used to construct biological networks. DAVID database was used to perform Gene ontology (GO) and Kyoto Encyclopedia of Genes and Genomes (KEGG) enrichment analysis. Molecular docking was performed to further verify the internal relationship between the active ingredients and key targets.

Results

A total of 169 bioactive ingredients and 127 gene targets of XQLD were identified. The network analysis indicated that quercetin, kaempferol, stigmasterol, $\beta$-sitosterol, and luteolin may be candidate agents. The IL6, VEGFA, NFKBIA, ICAM1, VCAM1, PPARG, IRF1, CYP3A4, CYP1B1 and CYP1A1 could become potential drug targets. The KEGG suggested that PI3K-AKT, Estrogen, FoxO, MAPK, HIF-1 signaling pathway may play a significant role in treating asthma. Molecular docking showed that quercetin, kaempferol, stigmasterol, $\beta$-sitosterol, and luteolin combined well with IL6, VEGFA, PPARG, CYP3A4.

\section{Conclusion}

This study predicted the main ingredients, potential drug targets and pharmacological mechanism of XQLD on asthma from a new sight, as well as provided a promising approach for the research of chemical basis and pharmacology in Traditional Chinese medicine (TCM).

\section{Introduction}

Bronchial asthma (asthma for short) is a chronic airway inflammatory disease characterized by airway hyperresponsiveness and reversible airflow limitation, which is involved with a variety of inflammatory cells (eosinophils, mast cells, $T$ lymphocytes etc.) and inflammatory mediators[1]. Affecting more than 300 million people in the world, asthma has become the most common chronic respiratory disease all over the world and its morbidity is increasing year by year[2,3]. At present, there is a lack of perfect drugs for asthma. Glucocorticoids, bronchodilators and other anti-inflammatory drugs can only relieve asthma symptoms such as cough, wheezing and chest tightness, and cannot fundamentally solve the problem. Moreover, long-term application of glucocorticoids will lead to serious side effects[4]. Compared with the 
above therapies, Traditional Chinese medicine (TCM) is relatively safe, effective, economic and fewer side effects. Moreover, it can regulate the body function from the whole[5, 6], providing an alternative for asthma patients.

According to Chinese medicine, bronchial asthma is a kind of paroxysmal disease with cough and wheezing, which belongs to the categories of "Xiao Bing" and "Chuan Zheng". The treatment is mainly about warming lung, dispersing cold, reducing phlegm and relieving wheezing. Xiaoqinglong Decoction

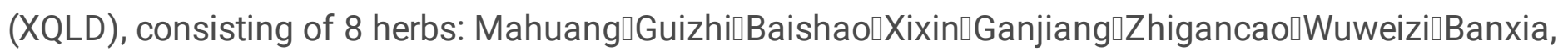
originates from Treatise on Febrile and Miscellaneous Diseases by famous Chinese physician by famous Chinese physician Zhang Zhongjing. It is one of the classical formula for asthma often used for "Han Xiao Zheng"[7]. The results of clinical trials show that the combination of western medicine and XQLD in the treatment of refractory asthma can significantly relieve cough, wheeze and other symptoms, improve

lung function and reduce toxic reactions and side effects and side effects caused by hormone therapy[8]. However, its underlying molecular mechanisms in curing asthma have not been clarified completely. And due to the multi-component, multi-target, and multi-pathway of Traditional Chinese medicine, it is difficult to understand the molecular mechanisms by conventional research methods. So new methods and strategies are needed to reveal its potential mechanism comprehensively and systematically. Network pharmacology, based on a variety of network data platforms, expounds the relationship between the active ingredients, targets, pathways and diseases from a system perspective by integrating pharmacology, molecular biology, systems biology and bioinformatics, providing a new method for indepth exploration of the mechanism of TCM $[9,10]$.

This study used systematic pharmacology and molecular docking methods to deeply explore the potential pharmacological effects of XQLD in the treatment of asthma, aiming to provide a solid scientific basis for the clinical application, and provide references for its in-depth research and further development and application. This method screened the effective ingredients of XQLD and potential targets for treating asthma, established a drug-component-target network, conducted Gene ontology (GO) and Kyoto Encyclopedia of Genes and Genomes (KEGG) enrichment analysis, and molecular docking was performed to verify the internal relationship between the active ingredients and core targets. The detailed process is shown in Fig. 1.

\section{Material And Methods}

\section{Screening Bioactive Components of XQLD}

Data on the 8 herbs in XQLD were mainly obtained from Traditional Chinese Medicine Systems Pharmacology Database and Analysis Platform (TCMSP, https://tcmspw.com/tcmsp.php),which is one of the largest Chinese medicine pharmacological data platforms including all Chinese herbal medicines, chemical components and pharmacokinetic properties information in the Pharmacopoeia of the People's Republic of China[11]. This study used oral bioavailability (OB) $>30 \%$ and drug-likeness (DL) $\geq 0.18$ as the screening criteria[12-14], the main active ingredients of XQLD were screened in TCMSP, and the 
compounds that did not meet the screening conditions but were high in content or had strong activity were selected and added manually by consulting the literature.

\section{The Putative Targets of XQLD}

According to the chemical similarities and pharmacophore models of the active ingredients screened, predict the target proteins of XQLD active ingredients in TCMSP. Import the predicted target protein names into the Uniprot (https://www.uniprot.org/) database with the species limited into "Homo sapiens" to obtain the gene symbols corresponding to the target proteins, that is the potential targets of the drug.

\section{Related targets of asthma}

Asthma-related targets were downloaded from OMIM database (http://www.omim.org/) and GeneCards database (https://www.genecards.org/) with the species limited into "Homo sapiens", "Asthma" and "bronchial asthma" as keywords were input OMIM and GeneCards database to obtain targets related to asthma.

\section{Construction of drug-compound-target network}

According to the correlation between drugs, active ingredients, and drug-related targets, Cytoscape 3.7.2 (http://www.cytoscape.org/) software was performed to construct drugs-compounds-targets network, aiming to visualize the direct or indirect correspondence between drugs, active ingredients and targets.

\section{Potential targets of XQLD for asthma}

Venny online platform 2.1 (https://bioinfogp.cnb.csic.es/tools/venny/) was used to integrate asthmatargets and drug-targets, the overlapping part is the potential target of XQLD for the treatment of asthma. Import the common target into the STRING online platform (https://string-db.Org/cgi/input.pl) to build a Protein-protein interaction (PPI) network. CytoHubba, a Cytoscape plugin, was performed to screen the top ten hub genes. At the same time, Cytoscape 3.7.2 software was used to construct compoundcommon target network of XQLD for asthma.

\section{Network topology analysis}

The internal regulation of the organism is often not dominated by a single signal pathway, but a complex regulatory network. PPI network is a network structure that connects proteins through molecular docking, which can be visually analyzed using the Bisogenet, a Cytoscape plugin. Bisogenet data are derived from the following six major PPI databases: Interacting Proteins (DIP), Biological General Repository for Interaction Datasets (BioGRID), Human Protein Reference Database (HPRD), IntAct Molecular Interaction Database(INTACT), Molecular INTeraction Database (MINT), and Biomolecular Interaction Network Database (BIND)[15]. In order to further study the molecular mechanism of XQLD in treating asthma, the common target of disease and drug was imported to Bisogenet with the species limited to "Homo Sapiens" to obtain proteins interaction relationship, and the CytoNCA plugin in Cytoscape was used to set the node central attributes value : Degree, Betweenness, Closeness, LAC, NC[16], screened the protein interaction relationship with high enrichment, and evaluated the core network 


\section{Enrichment analysis of GO and KEGG pathway}

In order to further explore the main biological processes and biological pathways of XQLD in treating asthma, GO and KEGG pathway enrichment analyses on the core network gene targets obtained above were conducted. 55 core targets were imported into the DAVID database (https://david.ncifcrf.gov/, Version 6.8), with the species limited to "Homo sapiens", threshold $P \leq 0.01$, and the results was visualized by $\mathrm{R}$ language software.

\section{Construction of target-pathway network}

Genes and proteins cannot exert their biological and pharmacological activities independently, but work through interaction networks and dynamic pathways at the cellular and molecular levels[17]. In order to further reveal the biological pathways of XQLD in treating asthma. Target-pathway network of XQLD for asthma was constructed according to the interaction relationship between pathways and genes, by using Cytoscape 3.7.2 software.

\section{Molecular docking}

According to hub gene, signal pathway and Previous research, the three-dimensional structure of IL-

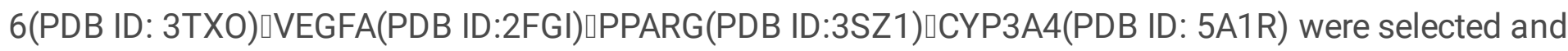
downloaded from RCSB PDB(Protein Data Bank) database (https://www.rcsb.org/) as the receptor for molecular docking. AutoDock Tools 1.5 .6 software was used to remove water molecule, separate proteins, add non-polar hydrogen, calculate the Gasteiger charge for these structures, and save it as a pdbqt file. The two-dimensional structure of the top 5 compounds(quercetin $\square$ kaempferol $\square$ Stigmasterol $\square$ beta-sitosterolluteolin) were obtained from the TCMSP database and convert them to pdbqt format as the docking ligand. The active site of molecular docking is determined by the ligand coordinates in the target protein complex. Virtual docking using Autodock Vina was performed to determine the binding affinity between protein models and the top 5 active compounds. Each docking result produced a total of 9 conformations, and the conformation with the smallest binding energy was selected for visual analysis in Pymol software.

\section{Results}

\section{Bioactive components and putative targets of XQLD}

A total of 1534 compounds of XQLD were retrieved from the TCMSP database. With OB > 30\% and DL > 0.18 as the screening criteria, we finally collected 169 main active ingredients of XQLD, of which there were 23, 7, 13, 8, 5, 92, 8 and 13 from Mahuang, Guizhi, Baishao, Xixin, Ganjiang, Zhigancao, Wuweizi and Banxia, respectively. The main compounds include: quercetin, kaempferol, beta-sitosterol, stigmasterol, luteolin and so on. According to the above-screened compounds, predicted the putative targets regulated by the active ingredients of XQLD, of which 8 compounds have no corresponding targets. After removing the duplicate targets, a total of 127 putative targets of XQLD were obtained. 


\section{Related targets of asthma}

We mainly obtain disease targets from the following two databases. 1). 173 asthma-related targets were obtained from the OMIM database; 2). 5649 candidate genes were preliminarily screened out from the Genecards database, and the top 400 results were selected to further study according to the "Relevance score". Integrating the targets obtained from the above two databases and removing duplicate targets, a total of 476 asthma-related targets were obtained.

\section{Drug- compound-target network analysis}

The drug-component-target network includes 268 nodes (133 compounds nodes, 127 target nodes, and 8 herbs nodes) and 1355 edges. The red triangles represent 8 herbs, the purple nodes represent the shared ingredients of the herbs, the green nodes represent the other ingredients, the yellow rectangles represent the putative targets, and edges represent the interaction between the herbs and the compounds or the compounds and the targets. Different herbs have the same active ingredients, and different active ingredients can regulate the same target, indicating that the different active ingredients of XQLD can play a synergistic effect when interacting. Showed in Fig. 2.

\section{Potential targets of XQLD for asthma}

A total of 32 common targets were obtained, that is the targets of XQLD for the treatment of asthma, Fig. 3A. The PPI network contains 32 nodes and 105 edges, Fig. 3B, among which IL6, VEGFA, NFKBIA, ICAM1, VCAM1, PPARG, IRF1, CYP3A4, CYP1B1, CYP1A1 possessed higher degrees and were more likely to be considered as the key target in treatment of XQLD for asthma, Fig. 3C. The Compound-Common target network of XQLD for asthma includes 146 nodes (32 common target nodes, 114 compound nodes) and 274 edges. Pink represents the compound, blue represents the common target, and edges represents the interaction between the active ingredients and the targets, Fig. 4. The top 5 degree of compounds of this network are quercetin, kaempferol, stigmasterol, beta-sitosterol, and luteolin. It is of great significance to the treatment of asthma. Basic information was shown in Table 1. 
Table 1

Basic information of the top five degree of the compounds

\begin{tabular}{|c|c|c|c|c|c|}
\hline MOL ID & Compound & Degree & OB & DL & Molecular formula \\
\hline MOL000098 & Quercetin & 22 & $46.43 \%$ & 0.28 & $\begin{array}{l}\text { 2-(3,4-Dihydroxyphenyl)-3,5,7- } \\
\text { trihydroxychromen-4-one }\end{array}$ \\
\hline MOL000422 & Kaempferol & 15 & $41.88 \%$ & 0.24 & $\begin{array}{l}\text { 3,5,7-Trihydroxy-2-(4- } \\
\text { hydroxyphenyl)chromen-4-one }\end{array}$ \\
\hline MOL000449 & $\begin{array}{l}\text { Beta- } \\
\text { sitosterol }\end{array}$ & 9 & $43.83 \%$ & 0.76 & $\begin{array}{l}\text { 3S,8S,9S,10R,13R,14S,17R)-17-[(2R,5S)-5- } \\
\text { ethyl-6-methylhept-3-en-2-yl]-10,13- } \\
\text { dimethyl-2,3,4,7,8,9,11,12,14,15,16,17- } \\
\text { dodecahydro-1H- }\end{array}$ \\
\hline MOL000358 & $\begin{array}{l}\text { Beta- } \\
\text { sitosterol }\end{array}$ & 9 & $36.91 \%$ & 0.75 & eta-sitosterol 3-0-glucoside_qt \\
\hline MOL000006 & luteolin & 7 & $36.16 \%$ & 0.25 & $\begin{array}{l}\text { 2-(3,4-dihydroxyphenyl)-5,7- } \\
\text { dihydroxychromen-4-one }\end{array}$ \\
\hline
\end{tabular}

\section{Network topology analysis}

In order to better understand the mechanism of XQLD in treating asthma, we adopted a network topology analysis method to evaluate the core network. Based on the protein-protein interaction relationship, common targets of XQLD and asthma were imported into Bisogenet, and the interactive PPI network of XQLD and asthma was initially obtained, Fig. 5A匹Then, the CytoNCA plugin was used to evaluate the core network with Degree $>60$, and got the first central network with 380 nodes and 14476 edges,

Fig. 5B.Finally, Degree $>101$, Betweenness $>160$, Closeness $>0.5, L A C>25, N C>60$ were set as criteria for secondary evaluation, after that, the second central network with 55 nodes and 705 edges was obtained, Fig. 5C. The detailed flowchart was shown in Fig. 5.

\section{GO and KEGG pathway enrichment analysis}

DAVID online platform was used to perform GO and KEGG enrichment analysis on 55 genes of the core network. GO enrichment analysis identified 921 items $(p<0.01)$, of which 790 items represent biological processes, mainly involved in regulation of DNA metabolic process, positive regulation of DNA-binding transcription factor activity, regulation of cell cycle phase transition, Response to topologically incorrect protein, post-translational protein modification, etc. 61 items represent cellular components, mainly refer to secretory granule lumen, ubiquitin ligase complex, vesicle lumen, melanosome, neuronal cell body, ficolin - 1-rich granule, distant axon, endoCytic vesicle, etc. 70 items represent molecular function, mainly involved in ubiquitin-like protein ligase binding, nuclear receptor binding, steroid hormone receptor binding, disordered domain specific binding, histone deacetylase binding, nuclear hormone receptor binding, p53 binding, RNA polymerase II transcription factor binding, general transcription initiation factor binding, MHC protein complex binding, etc. $\mathrm{GO}$ analysis results show that biological processes, cell components and molecular functions are closely related to the pathophysiological process of asthma. The results of 20 top-ranking were shown in Fig. 6. KEGG enrichment analysis obtained 63 signal 
pathways, including PI3K-Akt signaling pathway, Estrogen signaling pathway, Thyroid hormone signaling pathway, FoxO signaling pathway, MAPK signaling pathway, HIF-1 For signaling pathway, etc. The top 30 results of KEGG analysis are shown in Fig. 7.

\section{Target-pathway network analysis}

The target-pathway network consists of 66 nodes and 188 edges, including 30 KEGG signaling pathways and 36 genes. The red circle represents the pathway and the yellow rectangle represents the genes, edges represent the interaction between genes and pathway, the size of the node represents the degree of enrichment, Fig. 8. The result of this network showed that there existed complex interactions among multi-target and multi-signal pathway, which also played a synergistic effect on healing asthma.

\section{Results of molecular docking}

In molecular docking, if the ligand can combine with amino acid residue via some chemical bonds(such as $\mathrm{H}$ bond, $\mathrm{H}-\pi$ bond or $\pi-\pi$ bond)in the active pocket of the receptor and produces a negative binding energy which involving the process of energy complementation and conformational change, then the small molecule ligand and the receptor can form a stable complementation[18]. The more negative docking energy score, the higher binding ability and stability of ligand-receptor[19]. The results of molecular docking in this study show that small molecular ligands selected for docking can bind to the four key target proteins with lower binding energy, except the docking result of beta-sitosterol and PPARG $(-3.6 \mathrm{kcal} \cdot \mathrm{mol}-1)$ was not very satisfying (Table 2 and Fig. 9). Quercetin interacts with PPARG via two $\mathrm{H}$ Bond on HIS-266 (3.1 $\AA$ ), MYR-478 (2.6 ̊) with the lowest binding energy $\left(-10 \mathrm{kcal} \cdot \mathrm{mol}^{-1}\right)$, considered to be the most stable conformation (Fig. 9A). Quercetin formed three H bonds with ALA-564(3.2 $\AA$ ), GLU562(2.2 $\AA$ ), ASP-641(3.0 $)$ in VEGFA (Fig. 9C). CYP3A4 and quercetin were bound in LEU-211 (3.0 $)$ and ASP-214(3.0 A) by two $\mathrm{H}$ bonds Fig. 9B). Therefore,quercetin interacts with these amino acid residues through $\mathrm{H}$ bonds to remain a stable conformation. In addition, the PPARG-luteolin complex was stabilized in SER-342(3.5 $\AA$ ) and HIS-266(3.2 Å) by two H bonds (Fig. 9D). Luteolin interacted with VEGFA via four H

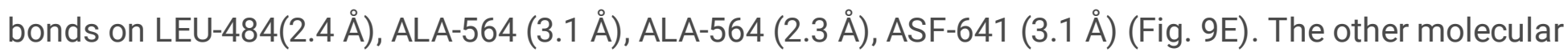
docking conformations can also produce negative binding energy through hydrophobic interaction, although no chemical bond was formed, indicating that the ligand-receptor complex has good binding ability and stability.

Table 2 Virtual docking of five bioactive ingredients from XQLD for asthma targets 


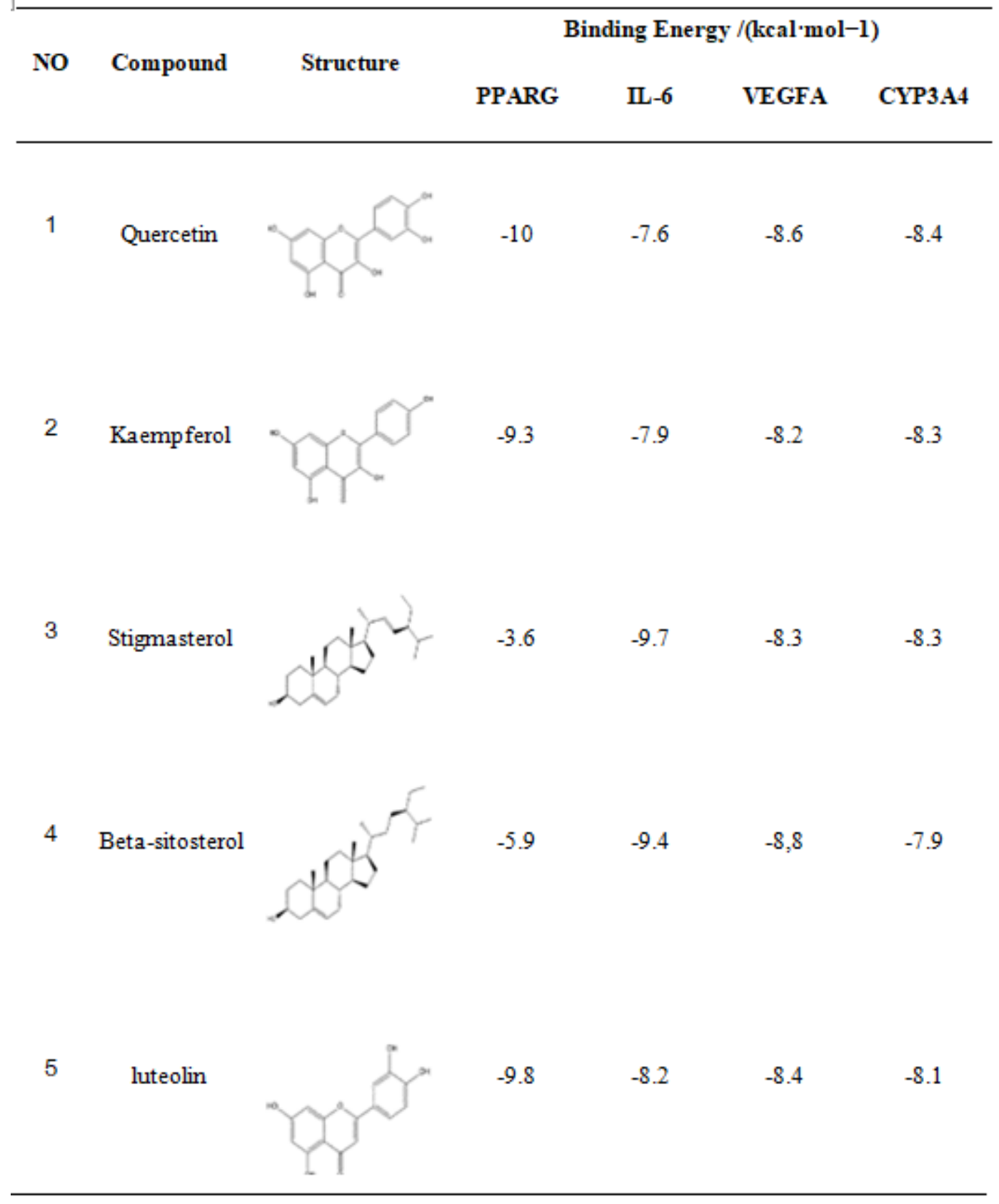

\section{Discussion}

A single drug component fundamentally targets a single molecular mechanism, so it may be difficult to effectively treat complex diseases with multicellular/histopathology[20, 21], such as asthma. Therefore, drugs that target multiple molecular signaling pathways are the focus of our future research. Traditional Chinese medicine characterized by multiple herbs and multiple components, working through multiple targets, multiple channels, and multiple biological process on diseases, it is difficult to fully clarify its mechanism by traditional research methods.

In this study, network pharmacology was performed to uncover the mechanism of XQLD in the treatment of asthma systematically, and the molecular docking was used to further verify the internal relationship between XQLD and asthma. The following aspects should attract our attention: 1) The drugscompounds-targets network showed that quercetin, kaempferol, stigmasterol, $\beta$-sitosterol and luteolin 
were more likely to be the key active components of XQLD in the treatment of asthma. 2) Compared with the common target PPI network, using the method of network topology analysis to obtain the core network could reveal the mechanism of XQLD in the treatment of asthma in detail. 3) The GO results showed that XQLD could regulate the treatment of asthma from the perspectives of genetics and epigenetics respectively. 4) The KEGG results showed that PI3K-Akt signaling pathway, Estrogen signaling pathway, FOXO signaling pathway, MAPK signaling pathway and HIF-1 signaling pathway were the key pathway in the treatment of asthma with XQLD. 5) The results of molecular docking show that there is a good binding ability between small molecular ligands and key target proteins.

The drug-compound-target network showed that quercetin, kaempferol, stigmasterol, $\beta$-sitosterol, and luteolin matched more targets, which are more likely to play a key role in treatment of XQLD for asthma. Among them, quercetin possessed the highest degree in this network, indicating that quercetin plays a key role in the treatment of asthma. Previous studies have shown that quercetin improves the inflammatory response and regulates the balance of $\mathrm{T} 1 / \mathrm{T} 2$ by inhibiting the production of inflammatory cytokines and chemokines[22-24]. Stigmasterol and kaempferol are able to maintain lung structure, reverse collagen deposition, and improve airway remodeling by reducing the proliferation and infiltration of inflammatory cells around bronchioles, blood vessels and alveoli[25]. Moreover, Kaempferol also possess the anti-inflammatory and antioxidant effects, which mediated by MAPK signal pathway and P38 signal pathway[26]. $\beta$-sitosterol and luteolin have been proven to alleviate airway inflammation and improve lung function through reducing the levels of cytoinflammatory factors such as TNFa, IL-4 and IL$5[27,28]$. Luteolin can also target PI3K-Akt signaling pathway to significantly inhibit the proliferation of vascular endothelial cells induced by VEGF[29]. Notably, there is a close synergistic effect among the effective components of XQLD, which can achieve the effect of treating asthma by reducing the proliferation and infiltration of inflammatory cells, reducing the level of inflammatory cytokines, delaying airway remodeling, improving lung function and so on.

By integrating of drug targets and disease targets, a total of 32 key targets of XQLD in the treatment of asthma were obtained. PPI network analysis of common targets suggested that IL6, VEGFA, NFKBIA, ICAM1, VCAM1, PPARG, IRF1, CYP3A4, CYP1B1 and CYP1A1 possessed higher degrees and showed a rich interaction with others, considered as hub gene in the treatment of asthma with XQLD. IL-6, a biomarker of systemic inflammation, participates in the process of inflammation and oxidative stress, and is positively correlated with the severity of asthma[30]. VEGFA, a member of the VEGF family, is a strong stimulator of allergic airway inflammation, airway remodeling and vascular leakage[31]. The activation of PPARG can moderate the initiation of inflammatory cells and pro-inflammatory cytokines, while allowing the expression of inflammatory cytokines in the lungs[32, 33]. On one hand, it can reduce airway inflammation, on the other hand, it can delay airway remodeling caused by chronic asthma[33]. Single nucleotide polymorphisms site variation in the promoter region of NFKBIA has been shown to alter susceptibility to infection and inflammation diseases, including asthma, viral bronchiolitis, and bronchopulmonary dysplasia[34]. CYP3A4, CYP1B1 and CYP1A1 are members of the cytochrome P450 (CYP) family, which participate in the biotransformation of most clinical drugs in vivo[35], and may regulate the pharmacokinetics of XQLD in vivo. VCAM1, a member of the immune globulin superfamily, 
interacts with integrin alpha-4/beta-1 on leukocytes, and mediates both adhesion and signal transduction, and may play an important pathophysiologic role both in immune responses and in leukocyte emigration to sites of inflammation (Uniprot). In order to further reveal the interaction mechanism between key active components and key target proteins, we carried out molecular docking. Four key targets (IL6, VEGFA, PPARG, CYP3A4) and five active components were analyzed by molecular docking. The results showed that a good visual model and strong binding affinity were formed between the active compound and the target protein. The screening of these specific index proteins can provide reliable detection indicators for further research on the clinical or animal experiments of XQLD in the treatment of asthma, which can effectively shorten the experimental period and reduce the experimental cost. However, how to strengthen the binding activity between the key active ingredients of XQLD and the key targets for the treatment of asthma to improve the therapeutic effect is the focus of future research.

GO enrichment analysis mainly involves positive regulation of DNA metabolic process, DNA/RNA transcription regulation, post-translational protein modification, nuclear receptor binding, histone deacetylase binding, etc., indicating that XQLD could regulate the treatment of asthma from the perspectives of genetics and epigenetics respectively. This is consistent with the view that asthma is the result of the interaction of genetic and environmental factors[36-38]. In bronchial biopsies of patients with asthma, histone acetyltransferase increased significantly and histone deacetylase activity decreased compared with normal respiratory tract[39]. previous research also confirmed that histone deacetylation can alleviate eosinophilic airway inflammation induced by asthma allergen[40]. Therefore, we have reason to speculate that up-regulating the activity of histone deacetylase is one of the mechanisms of XQLD in the treatment of asthma.

The results of KEGG enrichment analysis showed that XQLD was related to multiple signal pathways, among which the main signal pathways involved in the regulation of asthma were PI3KAktsignalingpathway, Estrogen signaling pathway, MAPK signaling pathway, FoxO signaling pathway and HIF-1 signalingpathway.PI3K-Akt signaling pathway possessed the highest enrichment degree and occupied a central position in the whole regulatory network, which interacts with other pathways to regulate the activation of inflammatory response cells and the release of inflammatory transmitters, and plays a crucial role in chronic inflammation. Phosphoinositide 3-kinase (PI3K) is a crucial signaling molecule, which plays an important role in many cellular and molecular mechanisms driving the pathophysiology of asthma, especially PI3K- $\delta$ (a member of class IA PI3Ks), which is closely related to allergic asthma induced by house dust mites. PI3K- $\delta$ gene knockout can significantly improve the symptoms of asthma induced by house dust mites[41]. PI3K inhibitors can effectively reduce the levels of inflammatory cytokines or inflammatory mediators such as IgE, IL-4, IL-5, IL-13, TNF- $a$ and IL-1 $\beta[42]$. Quercetin inhibits the PI3K-Akt signal pathway by inhibiting the expression of AKT1, thus reducing the release of inflammatory mediators, alleviating airway hyperresponsiveness and delaying airway remodeling[43]. Hypoxia inducible factor-1a (HIF-1a) plays an important role in immune and inflammatory response[44, 45]. Activation of HIF-1a during inflammation can enhance the expression of VEGF and promote airway angiogenesis in patients with asthma[46]. Quercetin can down-regulates the activity of PI3K-Akt signaling pathway to inhibit the expression of HIF-1, which moderates antigen- 
induced airway inflammation and hyperresponsiveness by regulating VEGF-mediated vascular leakage[ 47,48$]$. Regulatory $T$ (Treg) cells play an important role in inhibiting airway inflammation and airway hyperresponsiveness[49]. Mice lacking Treg cells, mainly show TH2-type pathology in the mucous membrane of the lung, which is characterized by allergic inflammation and asthma[50]. Forkhead box-0 (FOXO) transcription factors plays a key role in T cell differentiation, especially in regulatory $\mathrm{T}$ (Treg) cell differentiation[51]. FOXO transcription factor guides the differentiation of CD4 + T cells into Treg cells by inhibiting the PI3K-Akt signaling pathway in CD4 + T cells[52]. The deletion of FOXO1 or both FOXO1 and FOXO3 can seriously inhibit the development of Treg cells, and those developed cells are nonfunctional in vivo[51, 52]. Activation of MAPK signaling pathway can promote the production of inflammatory mediators and aggravate the clinical symptoms of allergic airway inflammation and asthma[53], while quercetin can inhibit the production of inflammatory cytokines and chemokines by blocking the activation of MAPK signaling pathway[54]. Studies have shown that the expression of estrogen receptors (ER) in the airway epithelial cells of asthmatic patients is increased. Compared with men, women have an increased incidence of asthma from adolescence, while hormone fluctuations in menstruation, pregnancy and menopause are related to changes in asthma symptoms. The results of animal experiments with sex hormone receptor gene knockout showed that estrogen signaling promotes various allergen-mediated airway inflammation, while androgens weaken[55].

\section{Conclusion}

Asthma has been the most common chronic respiratory disease in the world, of which the morbidity is increasing year by year. However, radical treatment drugs have not been discovered in treating asthma at present. XQLD is described as a commonly used drug for treatment and prevention of asthma for thousands of years. Our research aims to explore the mechanism of XQLD through network pharmacology and molecular docking and validate that XQLD plays a positive role in the treatment of asthma.127 drug-related targets were obtained from TCMSP database, 476 target genes of asthma were predicted via Genecards, OMIM databases and were mapped to targets of XQLD active ingredients. As a result, 32 common targets were selected as candidate targets for XQLD in treating asthma, furthermore, IL6, VEGFA, NFKBIA, ICAM1, VCAM1, PPARG, IRF1, CYP3A4, CYP1B1 and CYP1A1 might be the hub genes in treatment of XQLD for asthma. Although our research has examined the possible role of XQLD in treating asthma, we cannot confirm if such a mechanism really has an impact for this study mainly relies on database information, and only the number of interactions is considered in the network analysis, the strength of the interaction, the dosage of TCM, the decoction method and other issues are not considered. Therefore, the conclusions need to be further verified by experiments.

\section{Abbreviations}

XQLD, Xiaoqinglong Decoction; TCMSP, Traditional Chinese Medicine Systems Pharmacology Database and Analysis Platform; OB, oral bioavailability; DL, drug-likeness; TCM, Traditional Chinese medicine; PPI, protein-protein interaction; PDB, Protein Data Bank; BP, biological process; CC, cell composition; MF, 
molecular function; GO, Gene Ontology; KEGG, Kyoto Encyclopedia of Genes and Genomes; DIP, Interacting Proteins; BioGRID, Biological General Repository for Interaction Datasets; HPRD, Human Protein Reference Database; INTACT, IntAct Molecular Interaction Database; MINT, Molecular INTeraction Database; BIND, Biomolecular Interaction Network Database.

\section{Declarations}

\section{Acknowledgments}

The authors would like to thank the reviewers and also the authors of all references. The reviewer's advice really makes the great improvement of this paper.

\section{Authors' contributions}

Jingmin Deng and Haili Li conceived and designed the study. Jianpeng Zhou and Zuyou Wei collected the data. Haili Li and Feng Chen performed the data analysis, Jingmin Deng and Haili Li wrote the manuscript. All authors are responsible for reviewing data. All authors read and approved the final manuscript.

\section{Funding}

This work is supported by the Guangxi Provincial Natural Science Foundation (Grant No. 2017GXNSFAA198104)

\section{Availability of data and materials}

All data are available in the manuscript and they are showed in figures and tables.

\section{Ethics approval and consent to participate}

Not applicable.

\section{Consent for publication}

Not applicable.

\section{Competing interests}

The authors declare that there are no conflicts of interest in relation to this work.

\section{Author details}

${ }^{1}$ Department of Respiratory and Critical Care Medicine, The First Affiliated Hospital of Guangxi Medical University, Nanning, Guangxi 530021, People's Republic of China; ${ }^{2}$ Department of Orthopedics, Ruikang 
Hospital Affiliated to Guangxi University of Chinese Medicine, Nanning Guangxi 530011هPeople's Republic of China

\section{References}

1. Asthma GIF. GINA-2019-main-report-June-2019-wms. 2019.

2. Pavord ID, Beasley R, Agusti A, et al. After asthma: redefining airways diseases. LANCET. 2018;391(10118):350-400.

3. King-Biggs MB. Asthma. ANN INTERN MED. 2019;171(7):C49-C64.

4. McGregor MC, Krings JG, Nair P, et al. Role of Biologics in Asthma. Am J Respir Crit Care Med. 2019;199(4):433-445.

5. Qi J, Yu J, Tan Y, et al. Mechanisms of Chinese Medicine Xinmailong's protection against heart failure in pressure-overloaded mice and cultured cardiomyocytes. Sci Rep. 2017;7:42843.

6. Hao P, Jiang F, Cheng J, et al. Traditional Chinese Medicine for Cardiovascular Disease: Evidence and Potential Mechanisms. J AM COLL CARDIOL. 2017;69(24):2952-2966.

7. Xing C, Tian Z, Qingyan M, et al. Research Progress of Xiao Qinglong Decoction in Treatment of Bronchial Asthma. Hubei Univ TCM. 2018;20(04):121-125.

8. Wang L, Zheng X, Hui Y, et al. Adjuvant treatment with Xiaoqinglong formula for bronchial asthma: Protocol of systematic review and meta-analysis. Medicine (Baltimore). 2019;98(35):e17053.

9. Guo W, Huang J, Wang N, et al. Integrating Network Pharmacology and Pharmacological Evaluation for Deciphering the Action Mechanism of Herbal Formula Zuojin Pill in Suppressing Hepatocellular Carcinoma. FRONT PHARMACOL. 2019;10:1185.

10. Berger SI, lyengar R. Network analyses in systems pharmacology. BIOINFORMATICS. 2009;25(19):2466-2472.

11. Ru J, Li P, Wang J, et al. TCMSP: a database of systems pharmacology for drug discovery from herbal medicines. J Cheminform. 2014;6:13.

12. Wu W, Yang S, Liu P, et al. Systems Pharmacology-Based Strategy to Investigate Pharmacological Mechanisms of Radix Puerariae for Treatment of Hypertension. FRONT PHARMACOL. 2020;11:345.

13. Song W, Ni S, Fu Y, et al. Uncovering the mechanism of Maxing Ganshi Decoction on asthma from a systematic perspective: A network pharmacology study. Sci Rep. 2018;8(1):17362.

14. Lv X, Xu Z, Xu G, et al. Investigation of the active components and mechanisms of Schisandra chinensis in the treatment of asthma based on a network pharmacology approach and experimental 
validation. FOOD FUNCT. 2020;11(4):3032-3042.

15. Martin A, Ochagavia ME, Rabasa LC, et al. BisoGenet: a new tool for gene network building, visualization and analysis. BMC BIOINFORMATICS. 2010;11:91.

16. Tang Y, Li M, Wang J, et al. CytoNCA: a cytoscape plugin for centrality analysis and evaluation of protein interaction networks. BIOSYSTEMS. 2015;127:67-72.

17. Kumar A, Butler BM, Kumar S, et al. Integration of structural dynamics and molecular evolution via protein interaction networks: a new era in genomic medicine. Curr Opin Struct Biol. 2015;35:135-142.

18. Jian GH, Su BZ, Zhou WJ, et al. Application of network pharmacology and molecular docking to elucidate the potential mechanism of Eucommia ulmoides-Radix Achyranthis Bidentatae against osteoarthritis. BIODATA MIN. 2020;13:12.

19. Rahman N, Muhammad I, Khan H, et al. Molecular Docking of Isolated Alkaloids for Possible aGlucosidase Inhibition. Biomolecules. 2019;9(10).

20. Aggarwal BB, Sethi G, Baladandayuthapani V, et al. Targeting cell signaling pathways for drug discovery: an old lock needs a new key. J CELL BIOCHEM. 2007;102(3):580-592.

21. Kumar A, Zhang K. Advances in the Development of Shape Similarity Methods and Their Application in Drug Discovery. FRONT CHEM. 2018;6:315.

22. Cheng SC, Huang WC, S PJ, et al. Quercetin Inhibits the Production of IL-1 $\beta$-Induced Inflammatory Cytokines and Chemokines in ARPE-19 Cells via the MAPK and NF-KB Signaling Pathways. INT J MOL SCl. 2019;20(12).

23. Endale M, Park SC, Kim S, et al. Quercetin disrupts tyrosine-phosphorylated phosphatidylinositol 3kinase and myeloid differentiation factor-88 association, and inhibits MAPK/AP-1 and IKK/NF-KB-induced inflammatory mediators production in RAW 264.7 cells. IMMUNOBIOLOGY. 2013;218(12):1452-1467.

24. Zhu S, Wang $\mathrm{H}$, Zhang J, et al. Antiasthmatic activity of quercetin glycosides in neonatal asthmatic rats. 3 BIOTECH. 2019;9(5):189.

25. Antwi AO, Obiri DD, Osafo N. Stigmasterol Modulates Allergic Airway Inflammation in Guinea Pig Model of Ovalbumin-Induced Asthma. Mediators Inflamm. 2017;2017:2953930.

26. Huang X, Pan Q, Mao Z, et al. Kaempferol inhibits interleukin-1 $\beta$ stimulated matrix metalloproteinases by suppressing the MAPK-associated ERK and P38 signaling pathways. MOL MED REP. 2018;18(3):2697-2704.

27. Mahajan SG, Mehta AA. Suppression of ovalbumin-induced Th2-driven airway inflammation by $\beta$ sitosterol in a guinea pig model of asthma. EUR J PHARMACOL. 2011;650(1):458-464. 
28. Liang KL, Yu SJ, Huang WC, et al. Luteolin Attenuates Allergic Nasal Inflammation via Inhibition of Interleukin-4 in an Allergic Rhinitis Mouse Model and Peripheral Blood From Human Subjects With Allergic Rhinitis. FRONT PHARMACOL. 2020;11:291.

29. Bagli E, Stefaniotou M, Morbidelli L, et al. Luteolin inhibits vascular endothelial growth factor-induced angiogenesis; inhibition of endothelial cell survival and proliferation by targeting phosphatidylinositol 3'kinase activity. CANCER RES. 2004;64(21):7936-7946.

30. Peters MC, McGrath KW, Hawkins GA, et al. Plasma interleukin- 6 concentrations, metabolic dysfunction, and asthma severity: a cross-sectional analysis of two cohorts. Lancet Respir Med. 2016;4(7):574-584.

31. Lee CG, Link H, Baluk P, et al. Vascular endothelial growth factor (VEGF) induces remodeling and enhances TH2-mediated sensitization and inflammation in the lung. NAT MED. 2004;10(10):1095-1103.

32. Huang W, Glass CK. Nuclear receptors and inflammation control: molecular mechanisms and pathophysiological relevance. Arterioscler Thromb Vasc Biol. 2010;30(8):1542-1549.

33. Oh SH, Park SM, Lee YH, et al. Association of peroxisome proliferator-activated receptor-gamma gene polymorphisms with the development of asthma. Respir Med. 2009;103(7):1020-1024.

34. Ali S, Hirschfeld AF, Mayer ML, et al. Functional genetic variation in NFKBIA and susceptibility to childhood asthma, bronchiolitis, and bronchopulmonary dysplasia. J IMMUNOL. 2013;190(8):3949-3958.

35. Zanger UM, Schwab M. Cytochrome P450 enzymes in drug metabolism: regulation of gene expression, enzyme activities, and impact of genetic variation. Pharmacol Ther. 2013;138(1):103-141.

36. Schoettler N, Rodríguez E, Weidinger S, et al. Advances in asthma and allergic disease genetics: Is bigger always better? J Allergy Clin Immunol. 2019;144(6):1495-1506.

37. Ren Y, Su X, Kong L, et al. Therapeutic effects of histone deacetylase inhibitors in a murine asthma model. INFLAMM RES. 2016;65(12):995-1008.

38. Yang IV, Lozupone CA, Schwartz DA. The environment, epigenome, and asthma. J Allergy Clin Immunol. 2017;140(1):14-23.

39. Barnes PJ, Adcock IM, Ito K. Histone acetylation and deacetylation: importance in inflammatory lung diseases. EUR RESPIR J. 2005;25(3):552-563.

40. Zhang HP, Fu JJ, Fan T, et al. Histone deacetylation of memory T lymphocytes by You-Gui-Wan alleviates allergen-induced eosinophilic airway inflammation in asthma. Chin Med. 2015;10:9.

41. Kim SR, Park HJ, Lee KB, et al. Epithelial PI3K-ס Promotes House Dust Mite-Induced Allergic Asthma in NLRP3 Inflammasome-Dependent and -Independent Manners. Allergy Asthma Immunol Res. 
2020;12(2):338-358.

42. Kim JS, Jeong JS, Kwon SH, et al. Roles of PI3K pan-inhibitors and PI3K- $\delta$ inhibitors in allergic lung inflammation: a systematic review and meta-analysis. Sci Rep. 2020;10(1):7608.

43. Wan Y, Xu L, Liu Z, et al. Utilising network pharmacology to explore the underlying mechanism of Wumei Pill in treating pancreatic neoplasms. BMC Complement Altern Med. 2019;19(1):158.

44. Lee KS, Kim SR, Park SJ, et al. Peroxisome proliferator activated receptor-gamma modulates reactive oxygen species generation and activation of nuclear factor-kappaB and hypoxia-inducible factor 1alpha in allergic airway disease of mice. J Allergy Clin Immunol. 2006;118(1):120-127.

45. Li X, Shan C, Wu Z, et al. Emodin alleviated pulmonary inflammation in rats with LPS-induced acute lung injury through inhibiting the mTOR/HIF-1a/VEGF signaling pathway. INFLAMM RES. 2020;69(4):365373.

46. Meyer N, Akdis CA. Vascular endothelial growth factor as a key inducer of angiogenesis in the asthmatic airways. Curr Allergy Asthma Rep. 2013;13(1):1-9.

47. Kim SR, Lee KS, Park HS, et al. HIF-1 a inhibition ameliorates an allergic airway disease via VEGF suppression in bronchial epithelium. EUR J IMMUNOL. 2010;40(10):2858-2869.

48. Si LB, Zhang MZ, Han Q, et al. Sensitization of keloid fibroblasts by quercetin through the PI3K/Akt pathway is dependent on regulation of HIF-1a. AM J TRANSL RES. 2018;10(12):4223-4234.

49. Noval RM, Chatila TA. Regulatory T cells in allergic diseases. J Allergy Clin Immunol. 2016;138(3):639-652.

50. Josefowicz SZ, Niec RE, Kim HY, et al. Extrathymically generated regulatory T cells control mucosal TH2 inflammation. NATURE. 2012;482(7385):395-399.

51. Hedrick SM, Hess MR, Doedens AL, et al. FOXO transcription factors throughout T cell biology. NAT REV IMMUNOL. 2012;12(9):649-661.

52. Kerdiles YM, Stone EL, Beisner DR, et al. Foxo transcription factors control regulatory $\mathrm{T}$ cell development and function. IMMUNITY. 2010;33(6):890-904.

53. Alam R, Gorska MM. Mitogen-activated protein kinase signalling and ERK1/2 bistability in asthma. CLIN EXP ALLERGY. 2011;41(2):149-159.

54. Cheng SC, Huang WC, S PJ, et al. Quercetin Inhibits the Production of IL-1 $\beta$-Induced Inflammatory Cytokines and Chemokines in ARPE-19 Cells via the MAPK and NF-KB Signaling Pathways. INT J MOL SCl. 2019;20(12). 
55. Yung JA, Fuseini H, Newcomb DC. Hormones, sex, and asthma. Ann Allergy Asthma Immunol. 2018;120(5):488-494.

\section{Figures}

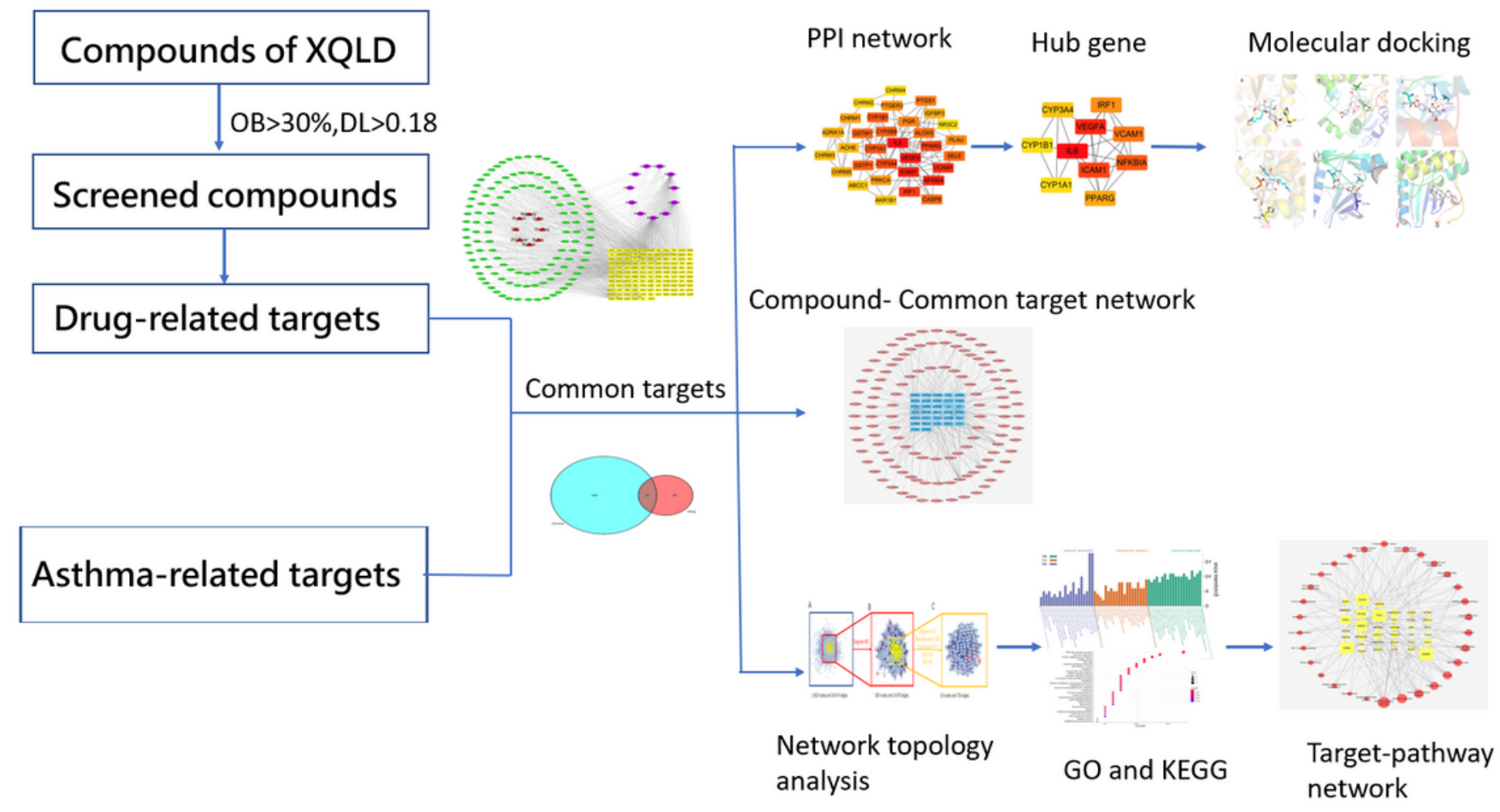

\section{Figure 1}

Workflow for XQLD on asthma. 


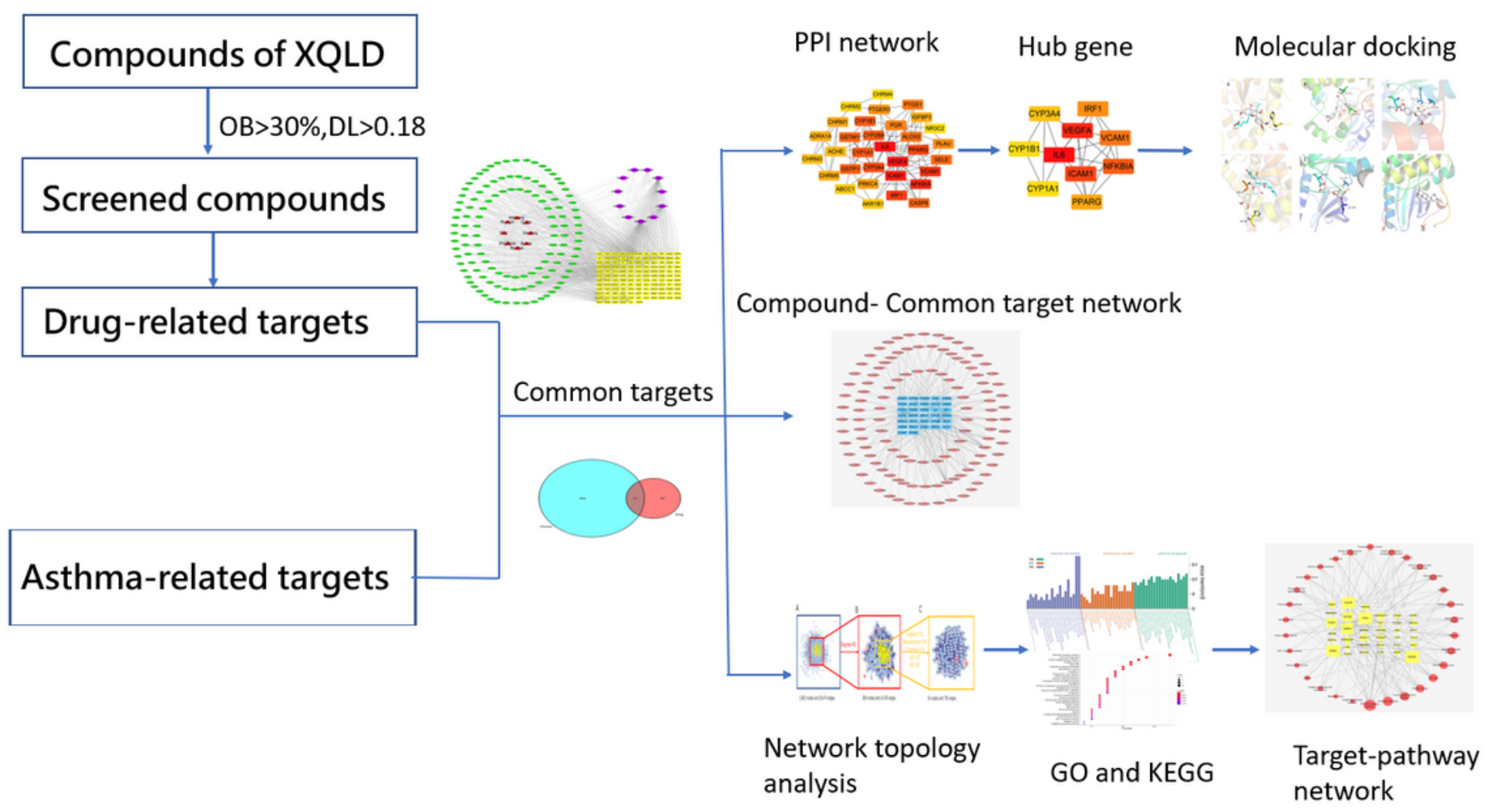

Figure 1

Workflow for XQLD on asthma.

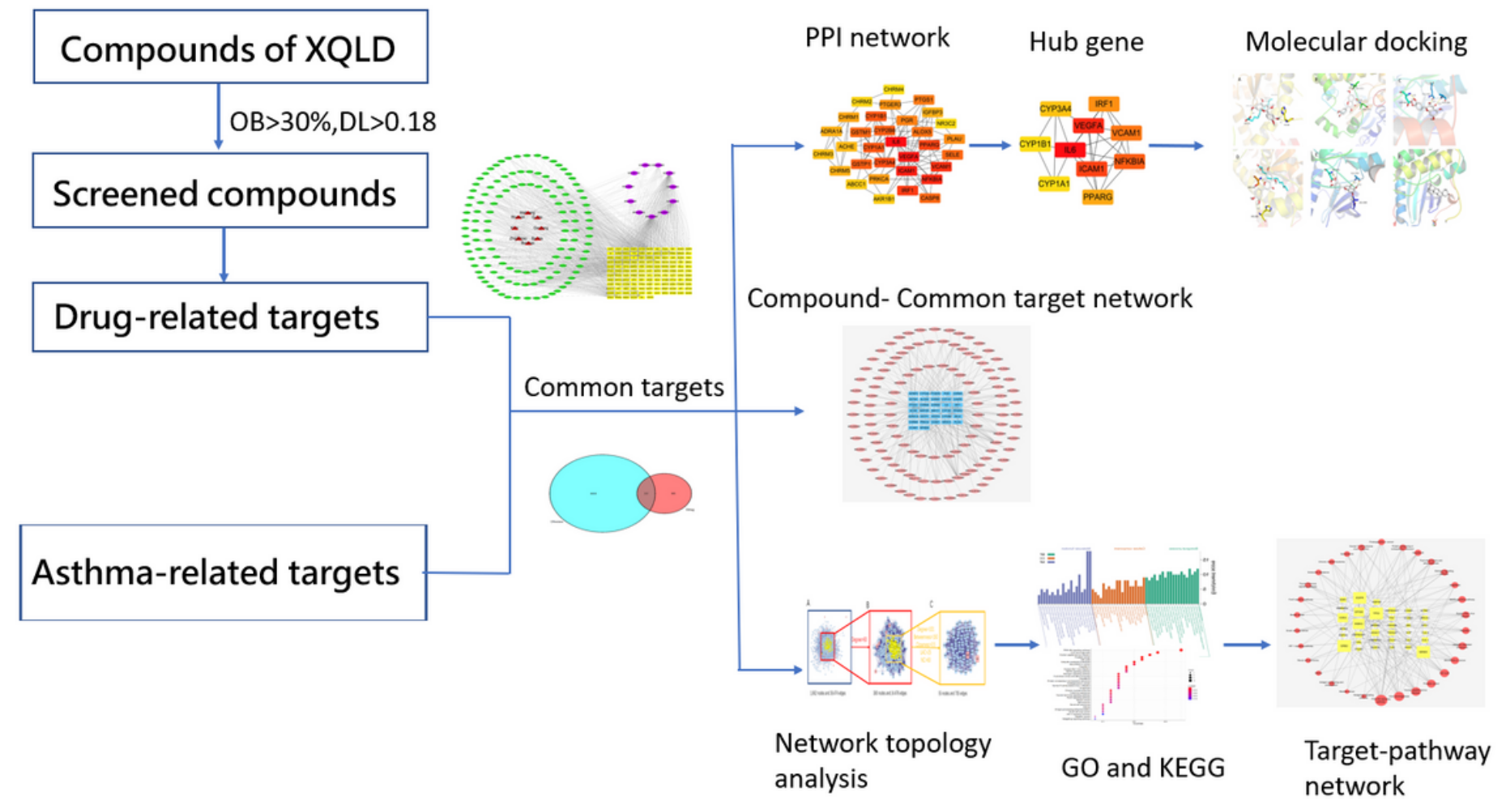

Figure 1 


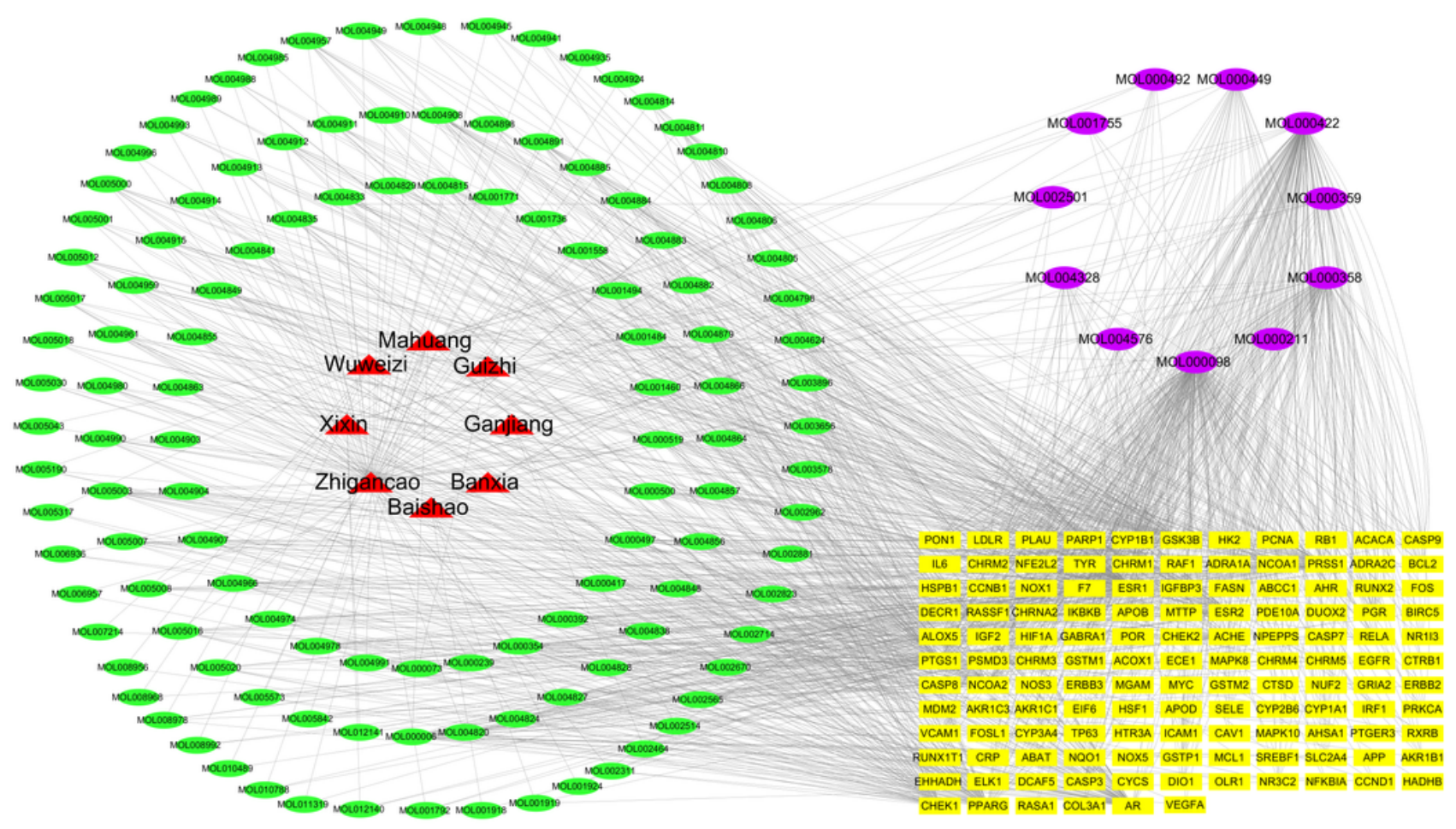

Figure 2

Drug-compound-target network. Red triangles represent 8 herbs, the purple nodes represent the shared ingredients of the herbs, the green nodes represent the other ingredients, the yellow rectangles represent the putative targets, edges represent the interaction between the nodes. 


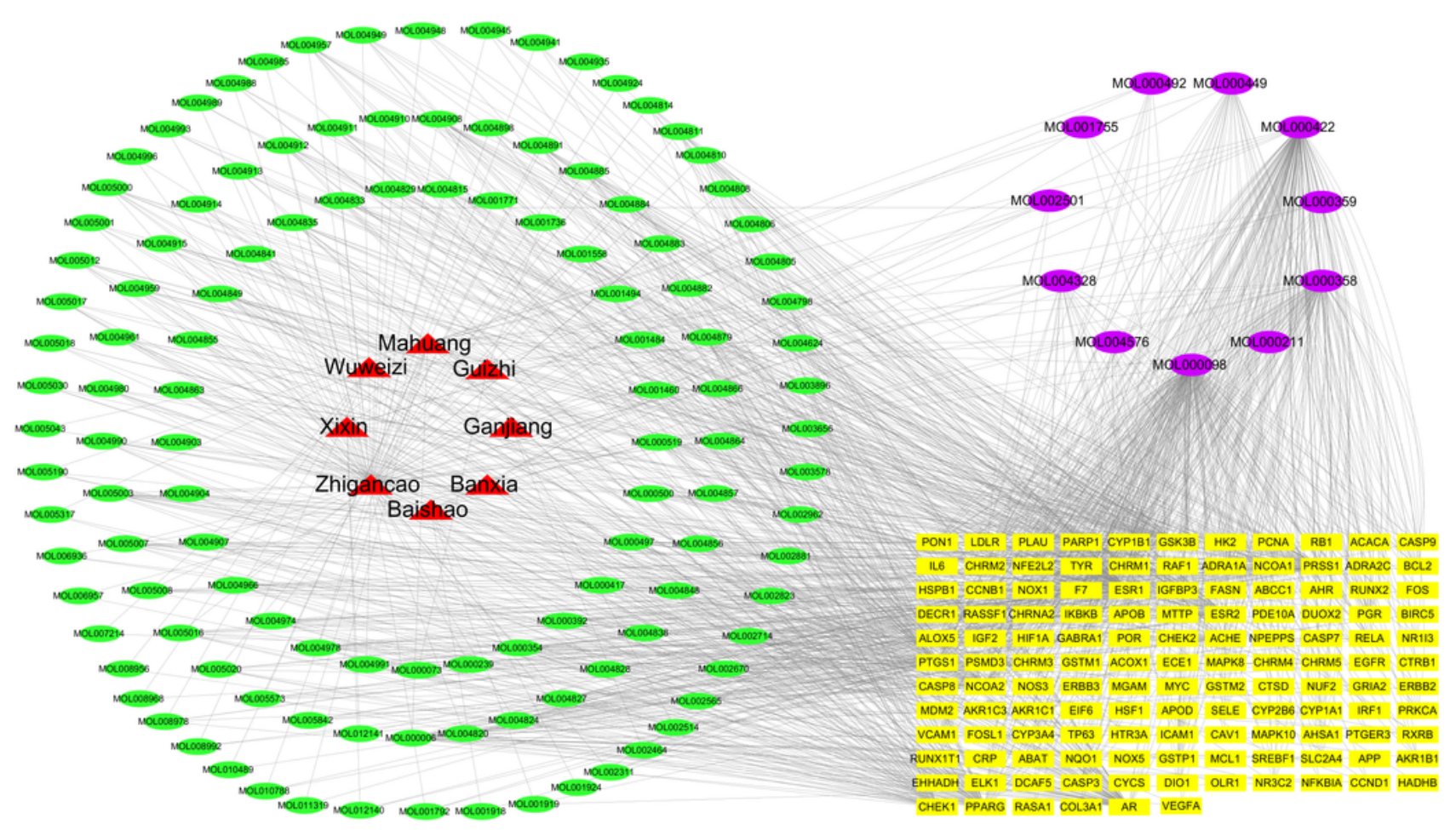

Figure 2

Drug-compound-target network. Red triangles represent 8 herbs, the purple nodes represent the shared ingredients of the herbs, the green nodes represent the other ingredients, the yellow rectangles represent the putative targets, edges represent the interaction between the nodes.

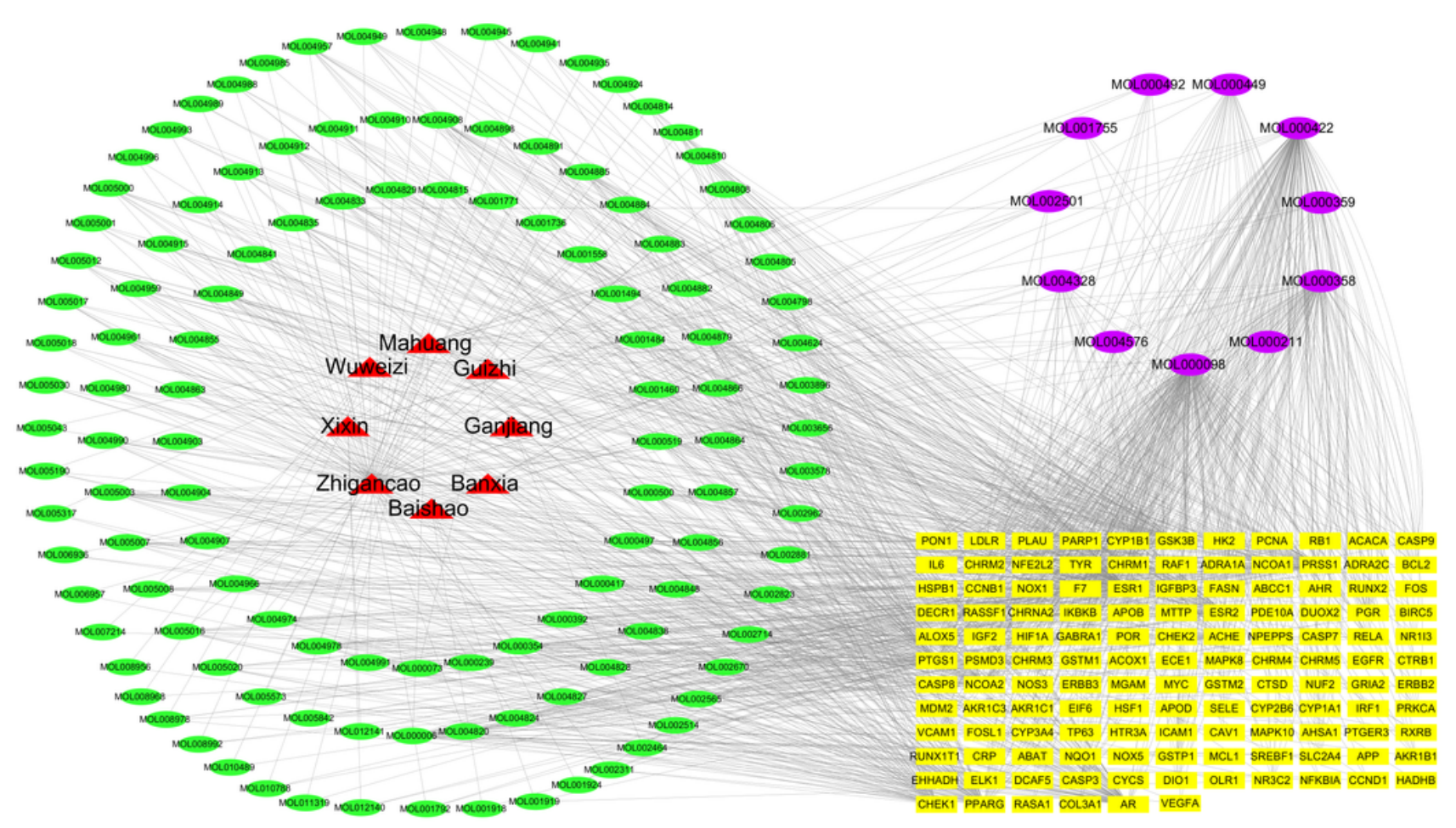

Page 21/41 
Figure 2

Drug-compound-target network. Red triangles represent 8 herbs, the purple nodes represent the shared ingredients of the herbs, the green nodes represent the other ingredients, the yellow rectangles represent the putative targets, edges represent the interaction between the nodes.

A

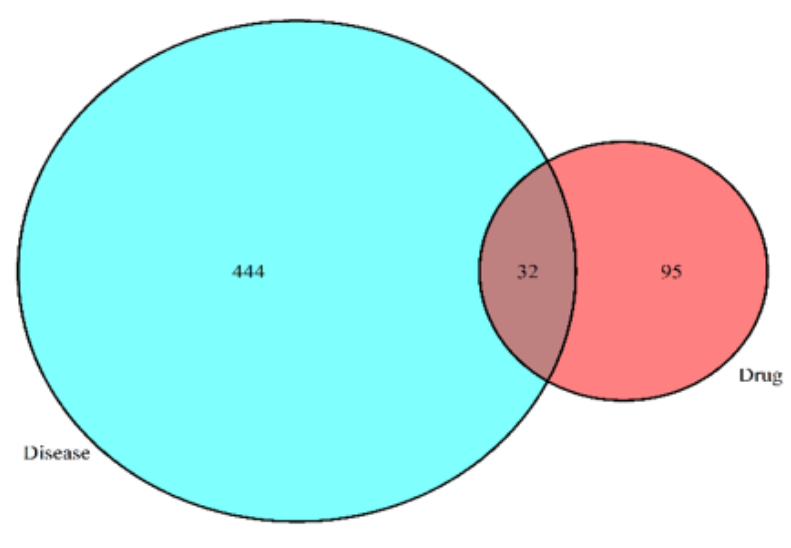

B

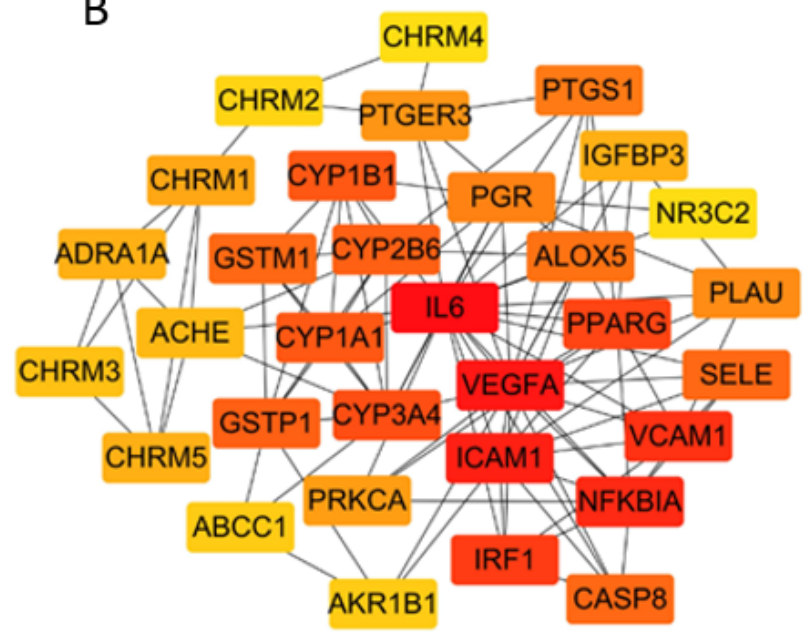

C

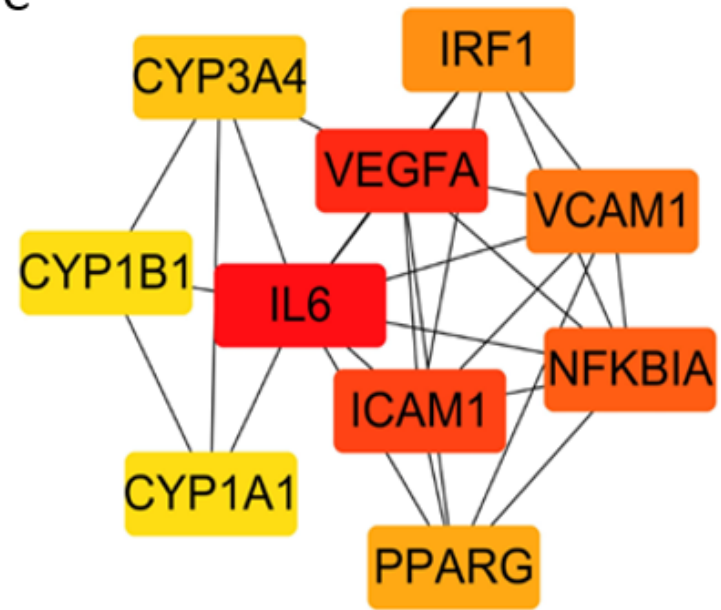

\section{Figure 3}

Potential targets of XQLD for asthma. (A) Venn diagram. Blue represents disease targets, red represents drug targets, and the overlapping part is 32 common targets. (B囚PPI network of common target, the redder the node color, the more significant it is in the PPI network. (C) The top10 degree genes in PPI network. 

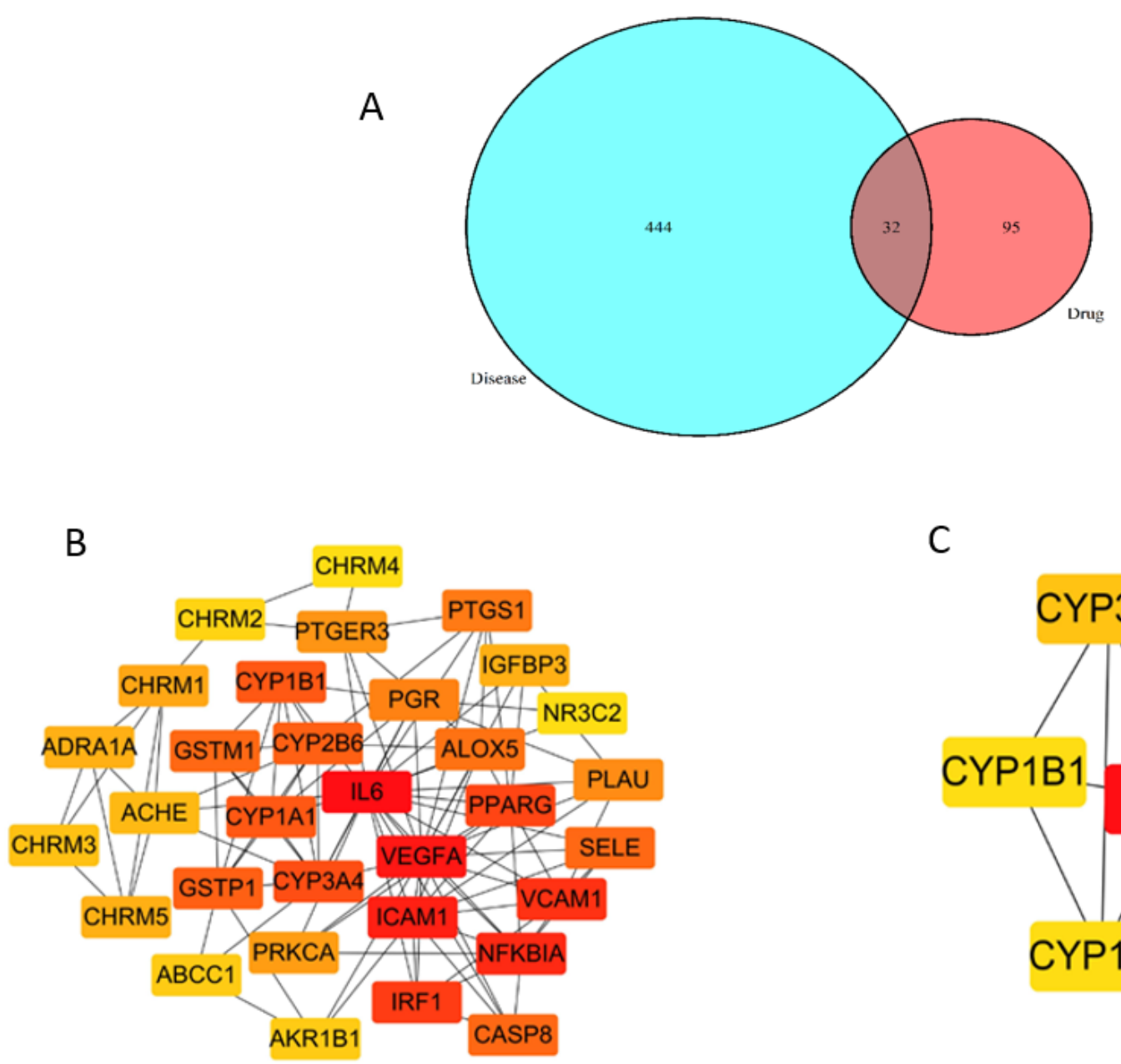

C

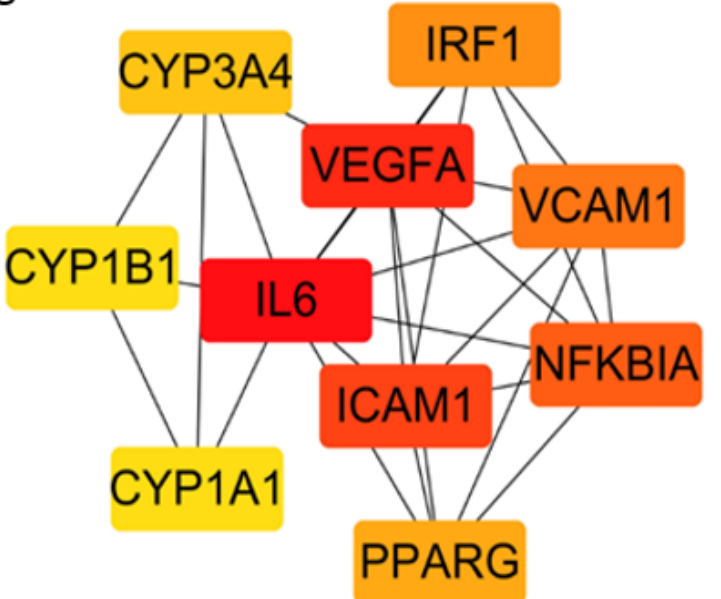

\section{Figure 3}

Potential targets of XQLD for asthma. (A) Venn diagram. Blue represents disease targets, red represents drug targets, and the overlapping part is 32 common targets. (B囚PPI network of common target, the redder the node color, the more significant it is in the PPI network. (C) The top10 degree genes in PPI network. 

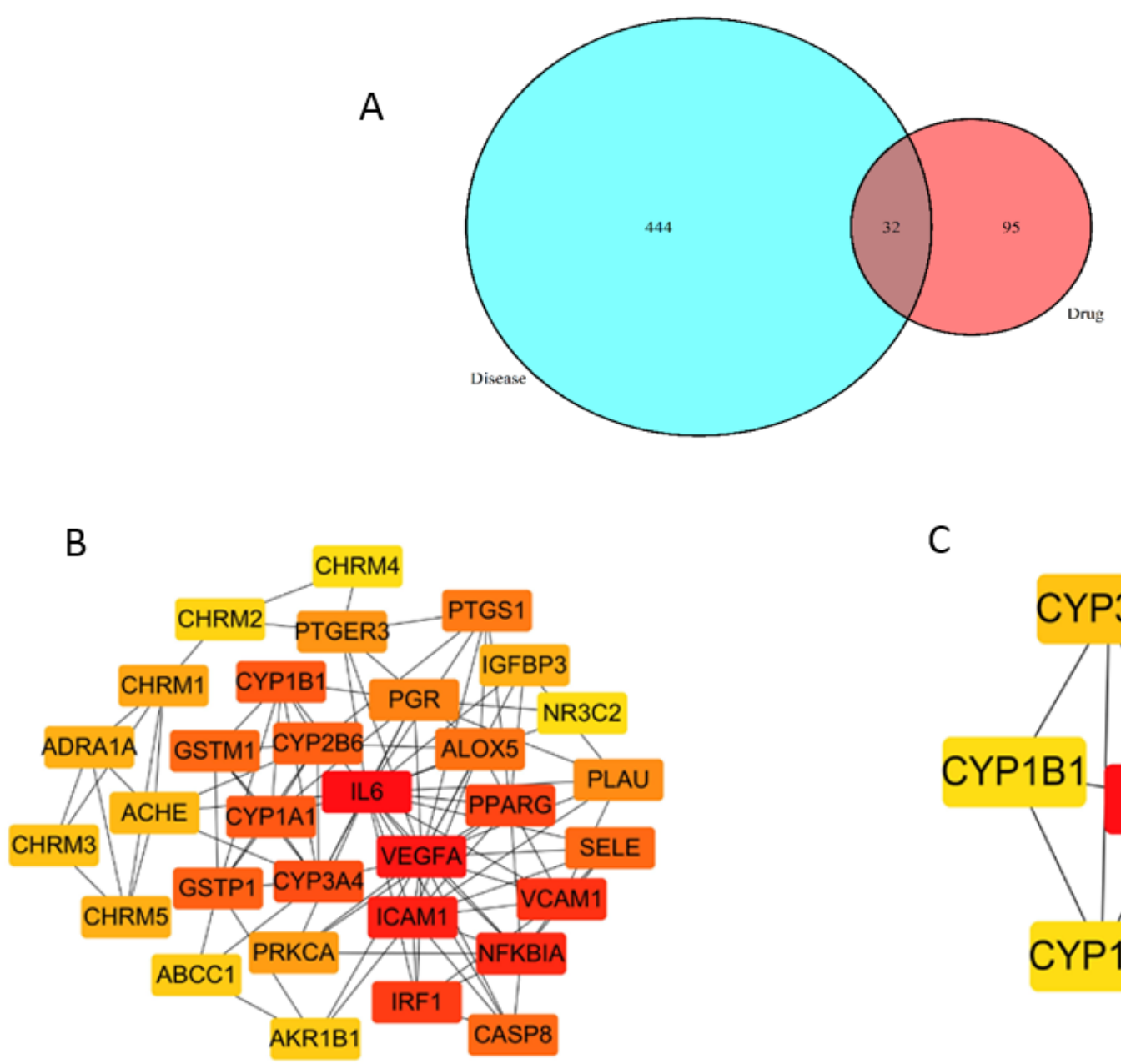

C

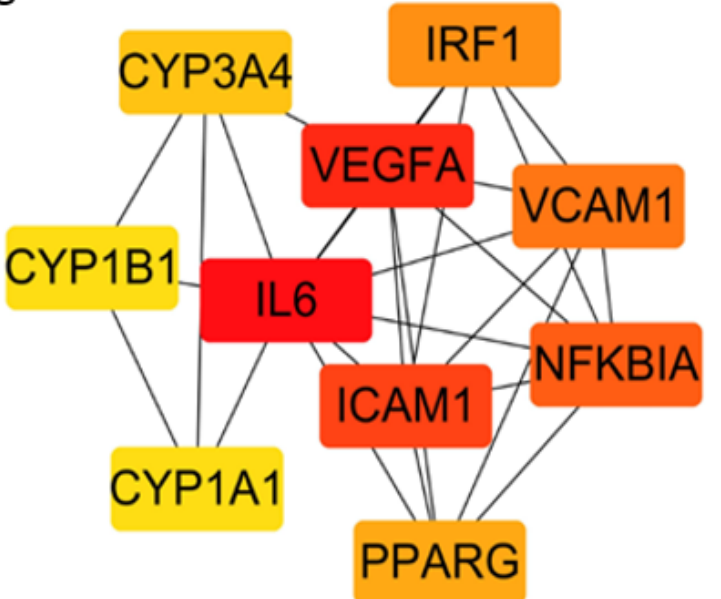

\section{Figure 3}

Potential targets of XQLD for asthma. (A) Venn diagram. Blue represents disease targets, red represents drug targets, and the overlapping part is 32 common targets. (B囚PPI network of common target, the redder the node color, the more significant it is in the PPI network. (C) The top10 degree genes in PPI network. 


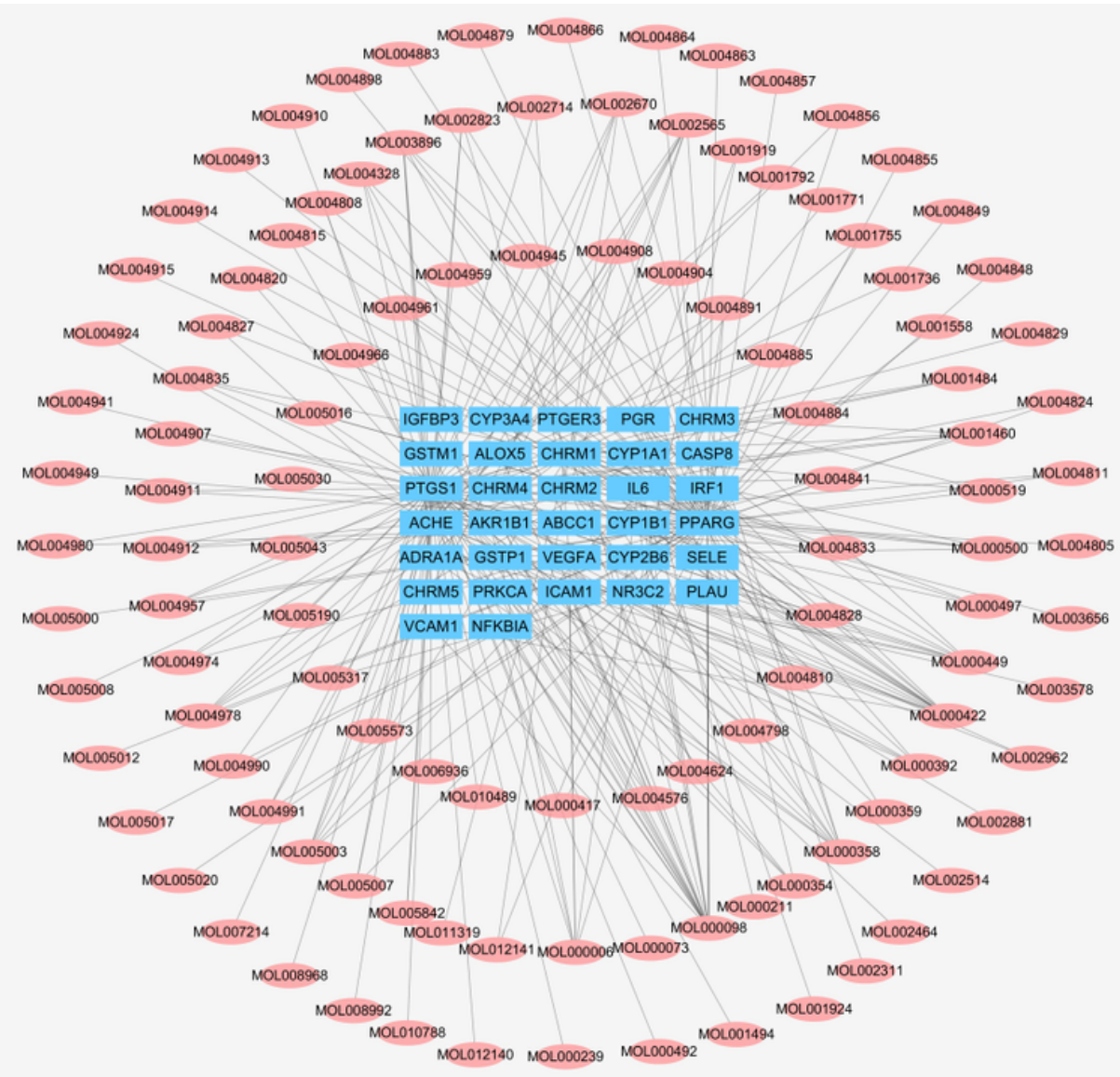

Figure 4

Compound-common target network of XQLD for asthma. The pink ovals represent the compounds, and the blue rectangles represent the common targets. 


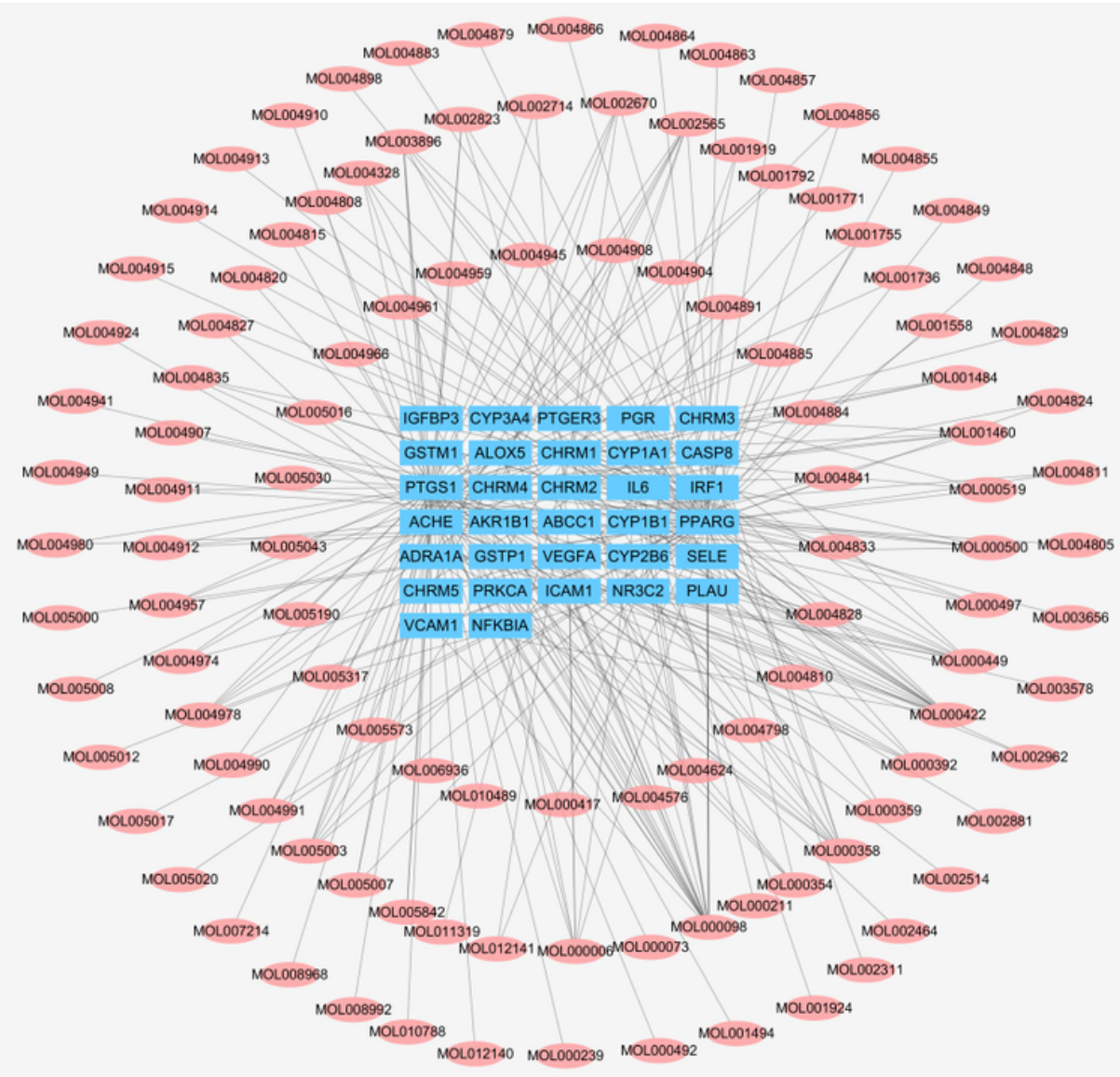

Figure 4

Compound-common target network of XQLD for asthma. The pink ovals represent the compounds, and the blue rectangles represent the common targets. 


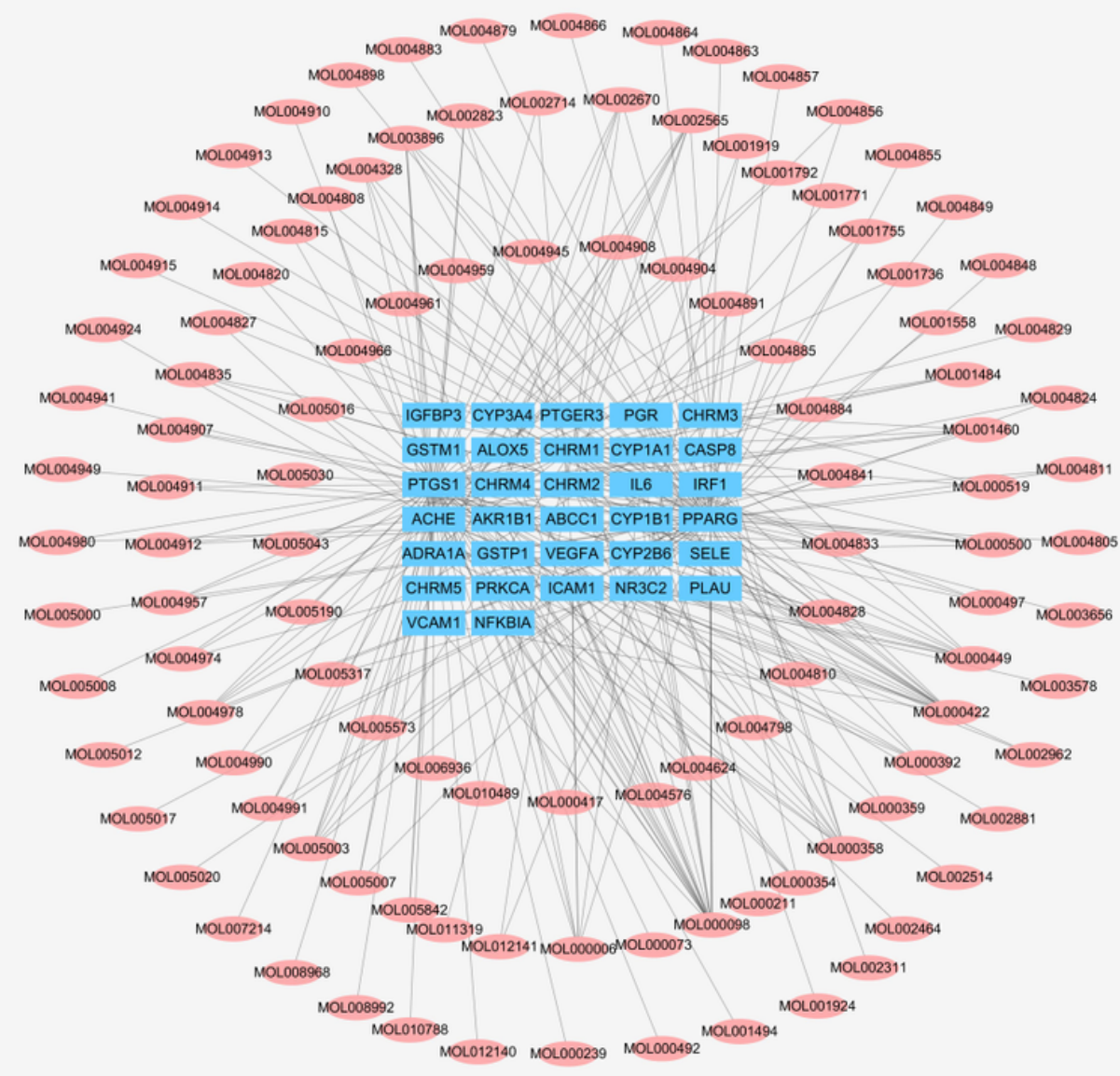

\section{Figure 4}

Compound-common target network of XQLD for asthma. The pink ovals represent the compounds, and the blue rectangles represent the common targets.

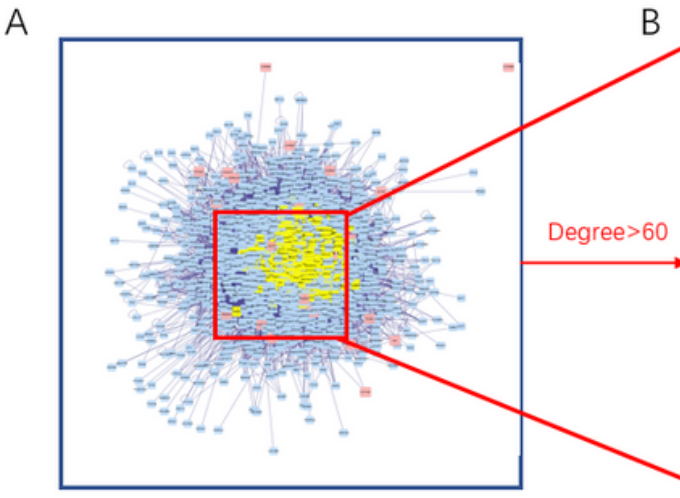

1,662 nodes and 36,474 edges

B

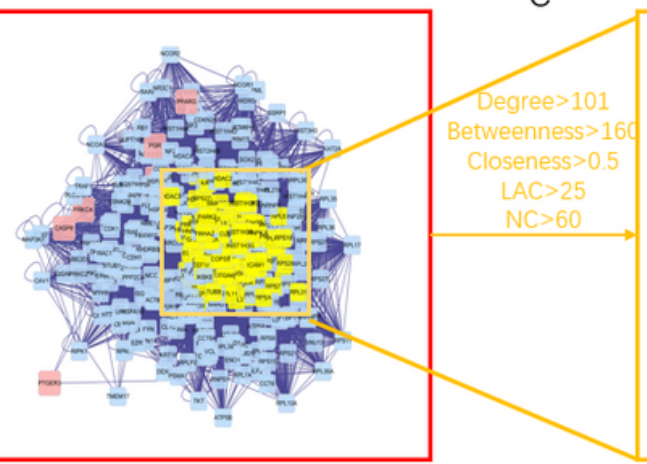

380 nodes and 14,476 edges

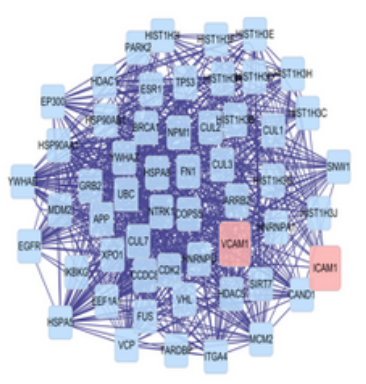

55 nodes and 705 edges

Figure 5 
Construction of XQLD-asthma PPI network. (A) Interactive PPI network of XQLD and asthma with 1662 nodes and 36474 edges. (B) First central network evaluation with a core subset of 380 nodes and 14476 edges based on the degree $>60$. (C) Second central network evaluation with a core subset of 55 nodes and 705 edges based on Degree $>101$ B Betweenness $>160 \square$ Closeness $>0.5 \square L A C>25 \square N C>60$. Blue diamonds, pink diamonds, and yellow diamonds stand for other human proteins, component targets, and selected targets, respectively.

A

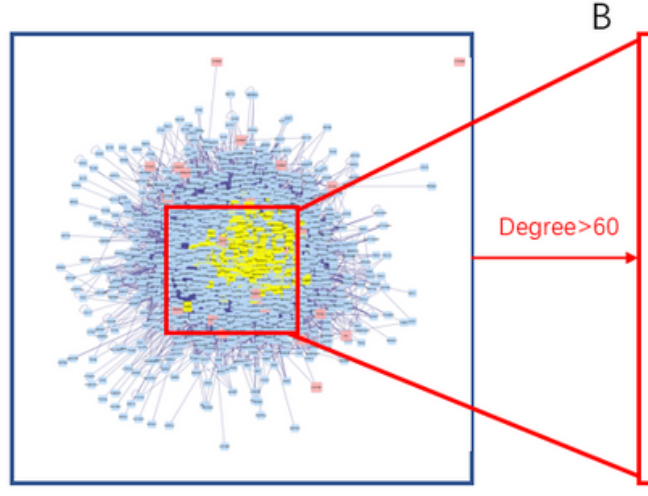

1,662 nodes and 36,474 edges

\section{$B$}

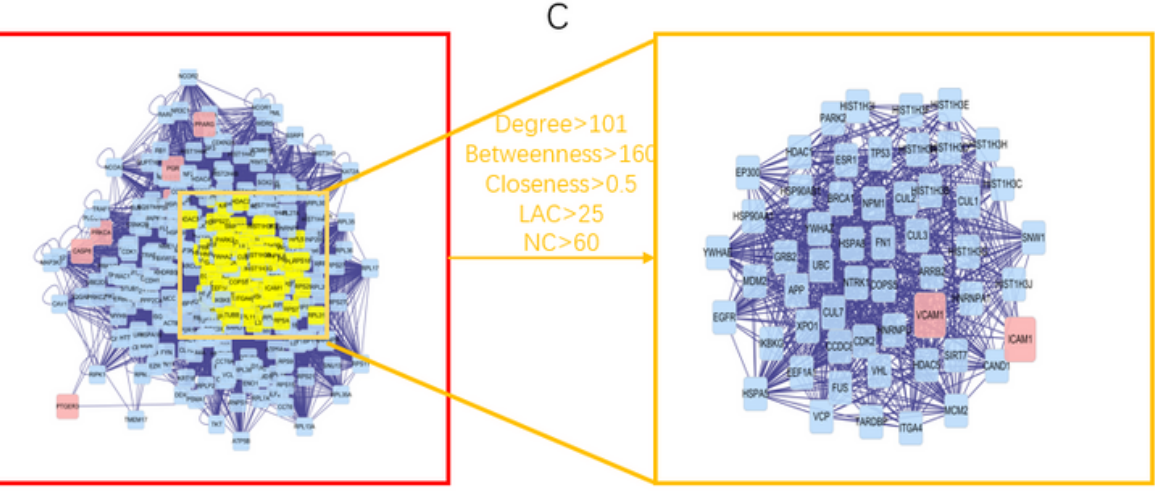

380 nodes and 14,476 edges

55 nodes and 705 edges

\section{Figure 5}

Construction of XQLD-asthma PPI network. (A) Interactive PPI network of XQLD and asthma with 1662 nodes and 36474 edges. (B) First central network evaluation with a core subset of 380 nodes and 14476 edges based on the degree $>60$. (C) Second central network evaluation with a core subset of 55 nodes and 705 edges based on Degree $>101$ Betweenness $>160 \square$ Closeness $>0.5 \square L A C>25 \square N C>60$. Blue diamonds, pink diamonds, and yellow diamonds stand for other human proteins, component targets, and selected targets, respectively. 


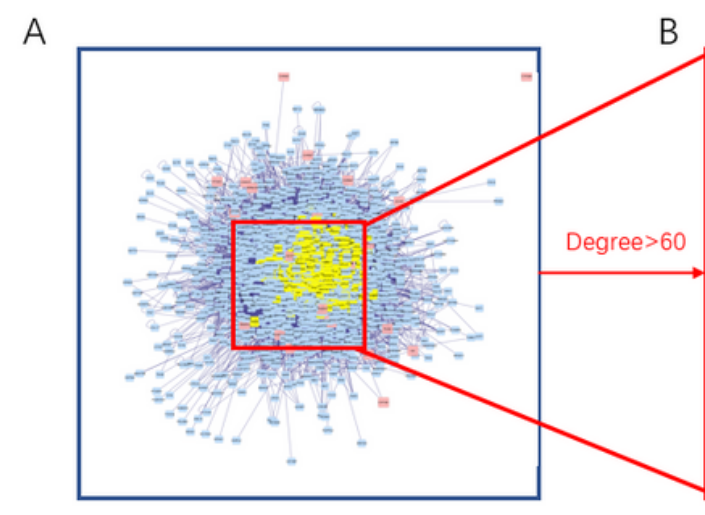

1,662 nodes and 36,474 edges

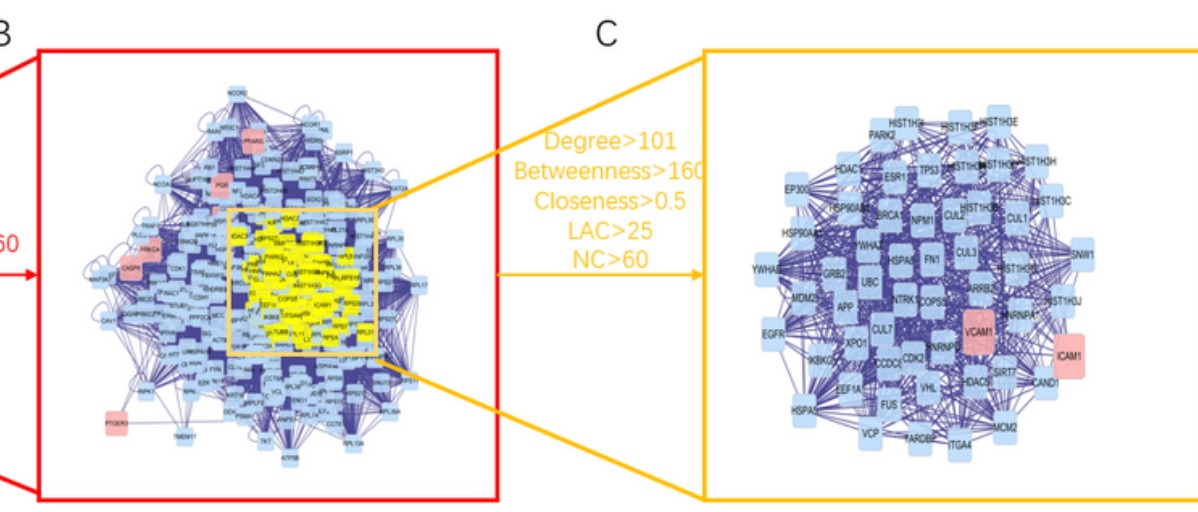

380 nodes and 14,476 edges
55 nodes and 705 edges

\section{Figure 5}

Construction of XQLD-asthma PPI network. (A) Interactive PPI network of XQLD and asthma with 1662 nodes and 36474 edges. (B) First central network evaluation with a core subset of 380 nodes and 14476 edges based on the degree $>60$. (C) Second central network evaluation with a core subset of 55 nodes and 705 edges based on Degree $>1010$ Betweenness $>160 \square$ Closeness $>0.5 \square L A C>25 \square N C>60$. Blue diamonds, pink diamonds, and yellow diamonds stand for other human proteins, component targets, and selected targets, respectively. 


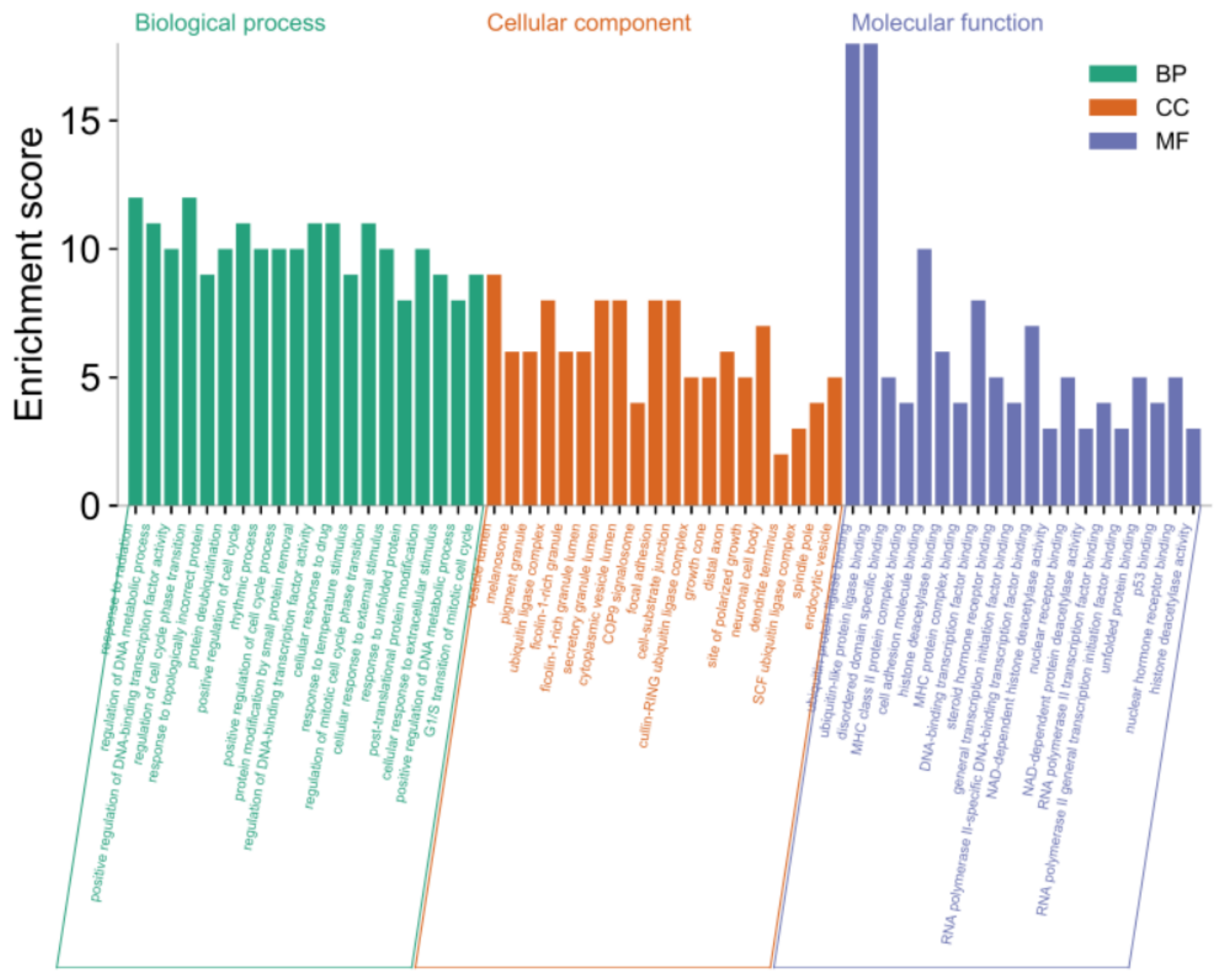

Figure 6

GO enrichment analysis of 55 targets of core network (the top 20 results. The abscissa represents the GO enrichment analysis item, and the ordinate represents the enrichment score. The higher the enrichment score, the more significant the enrichment analysis result. 


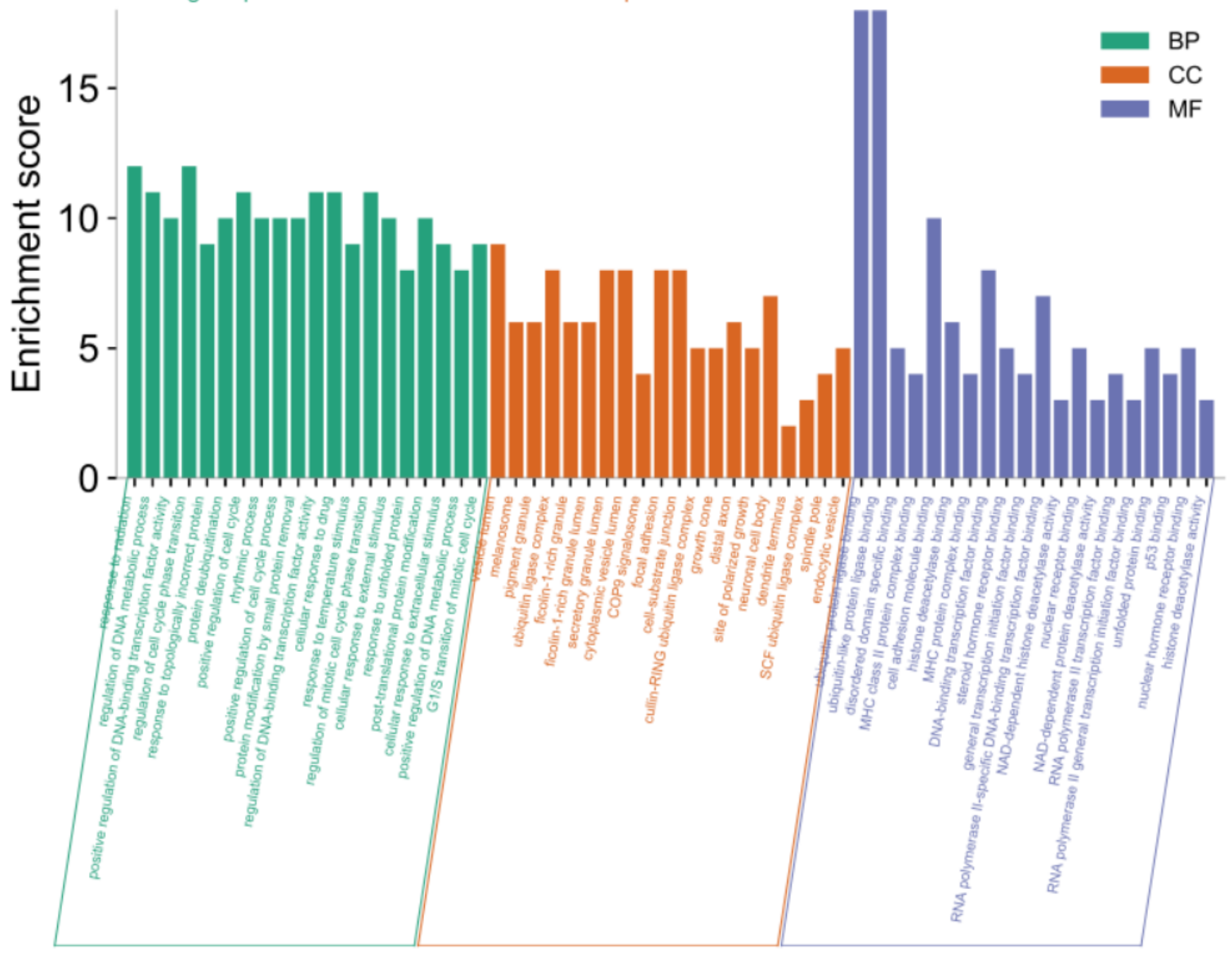

Figure 6

GO enrichment analysis of 55 targets of core network (the top 20 results. The abscissa represents the GO enrichment analysis item, and the ordinate represents the enrichment score. The higher the enrichment score, the more significant the enrichment analysis result. 


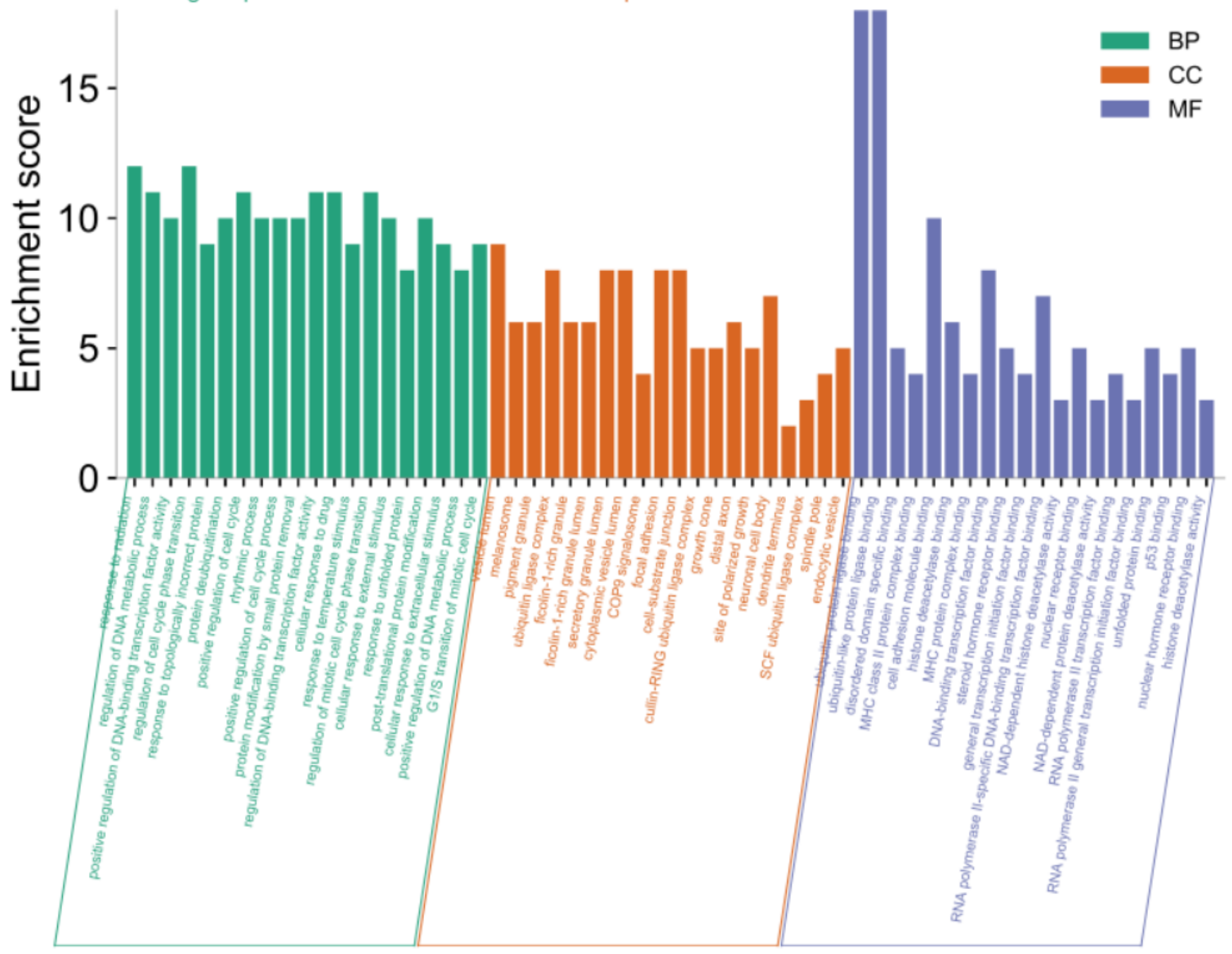

Figure 6

GO enrichment analysis of 55 targets of core network (the top 20 results. The abscissa represents the GO enrichment analysis item, and the ordinate represents the enrichment score. The higher the enrichment score, the more significant the enrichment analysis result. 


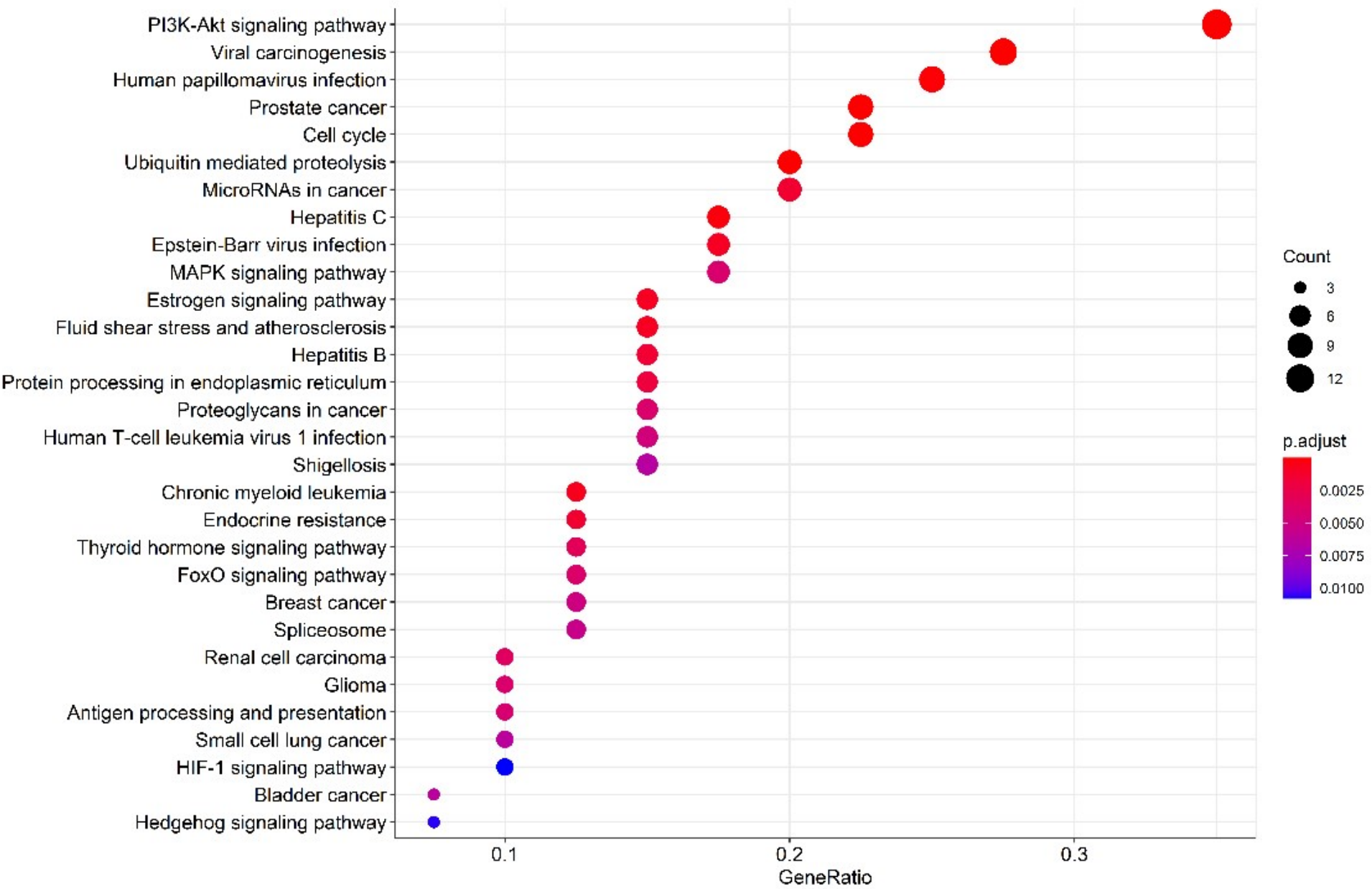

Figure 7

KEGG enrichment analysis of 55 targets of core network (the top 30 results). The ordinate represents the name of the pathway, and the abscissa represents GeneRatio. The size of the dots, and color of the dots represent the degree of KEGG enrichment analysis. 


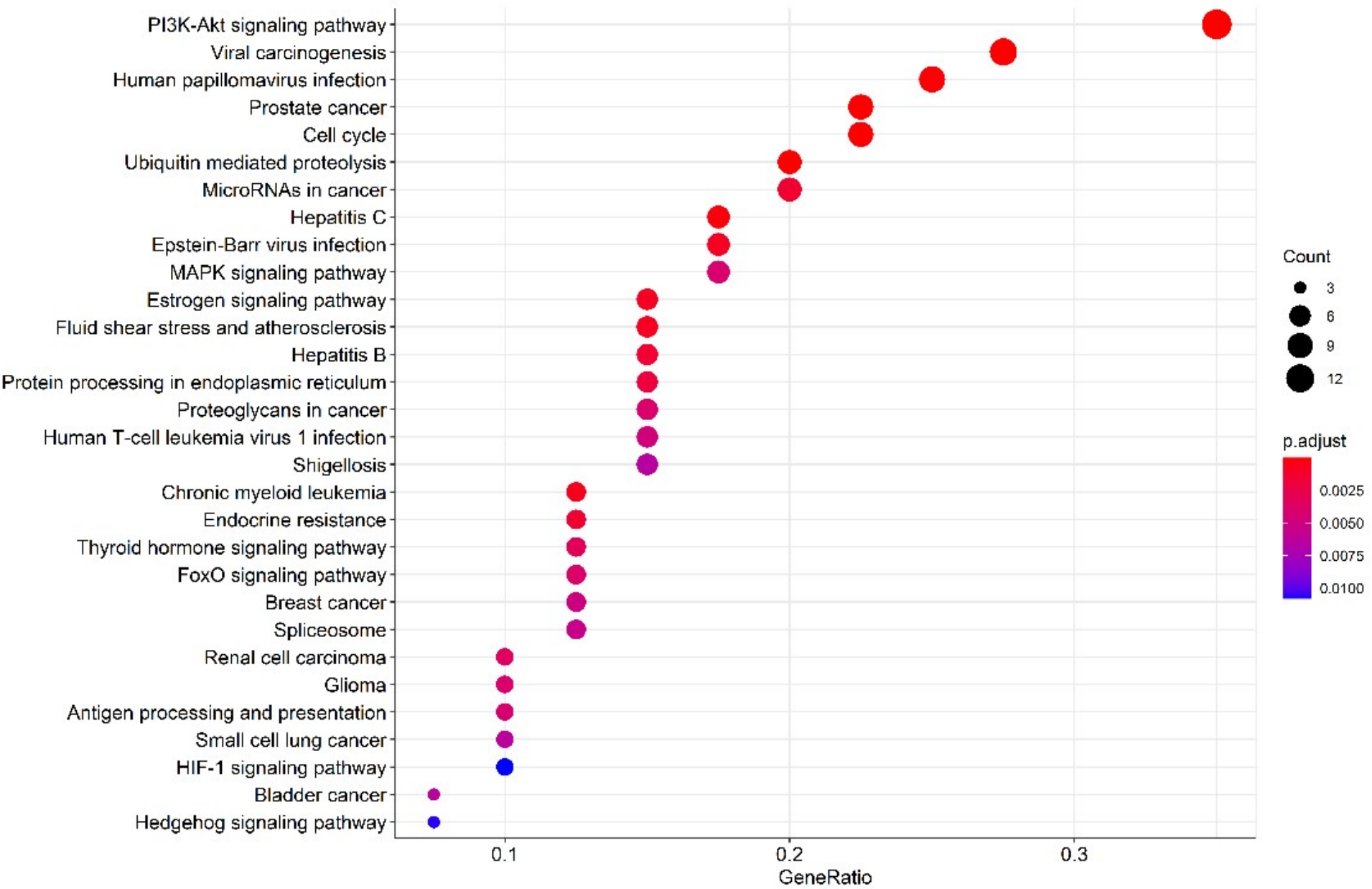

Figure 7

KEGG enrichment analysis of 55 targets of core network (the top 30 results). The ordinate represents the name of the pathway, and the abscissa represents GeneRatio. The size of the dots, and color of the dots represent the degree of KEGG enrichment analysis. 


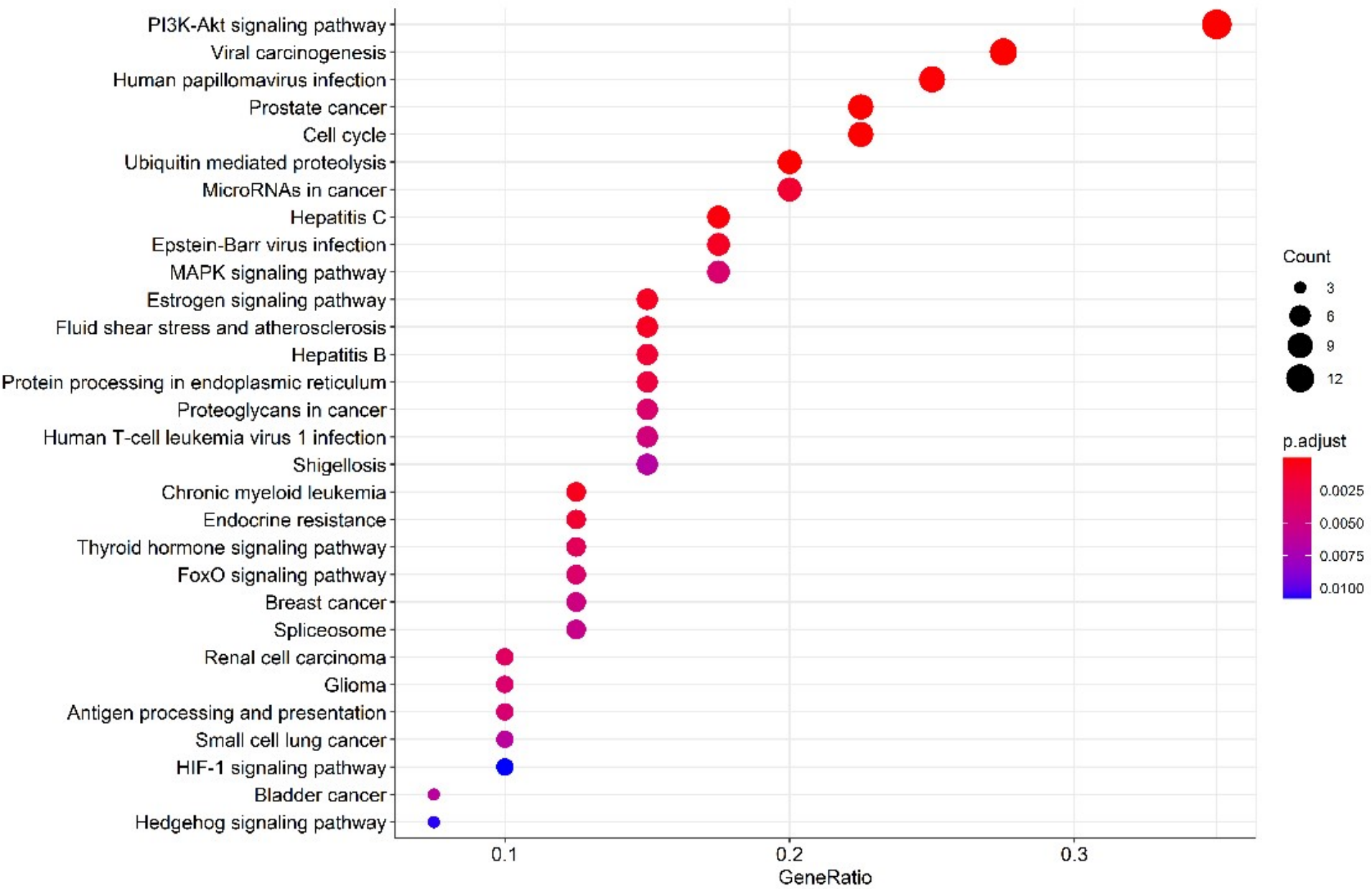

Figure 7

KEGG enrichment analysis of 55 targets of core network (the top 30 results). The ordinate represents the name of the pathway, and the abscissa represents GeneRatio. The size of the dots, and color of the dots represent the degree of KEGG enrichment analysis. 


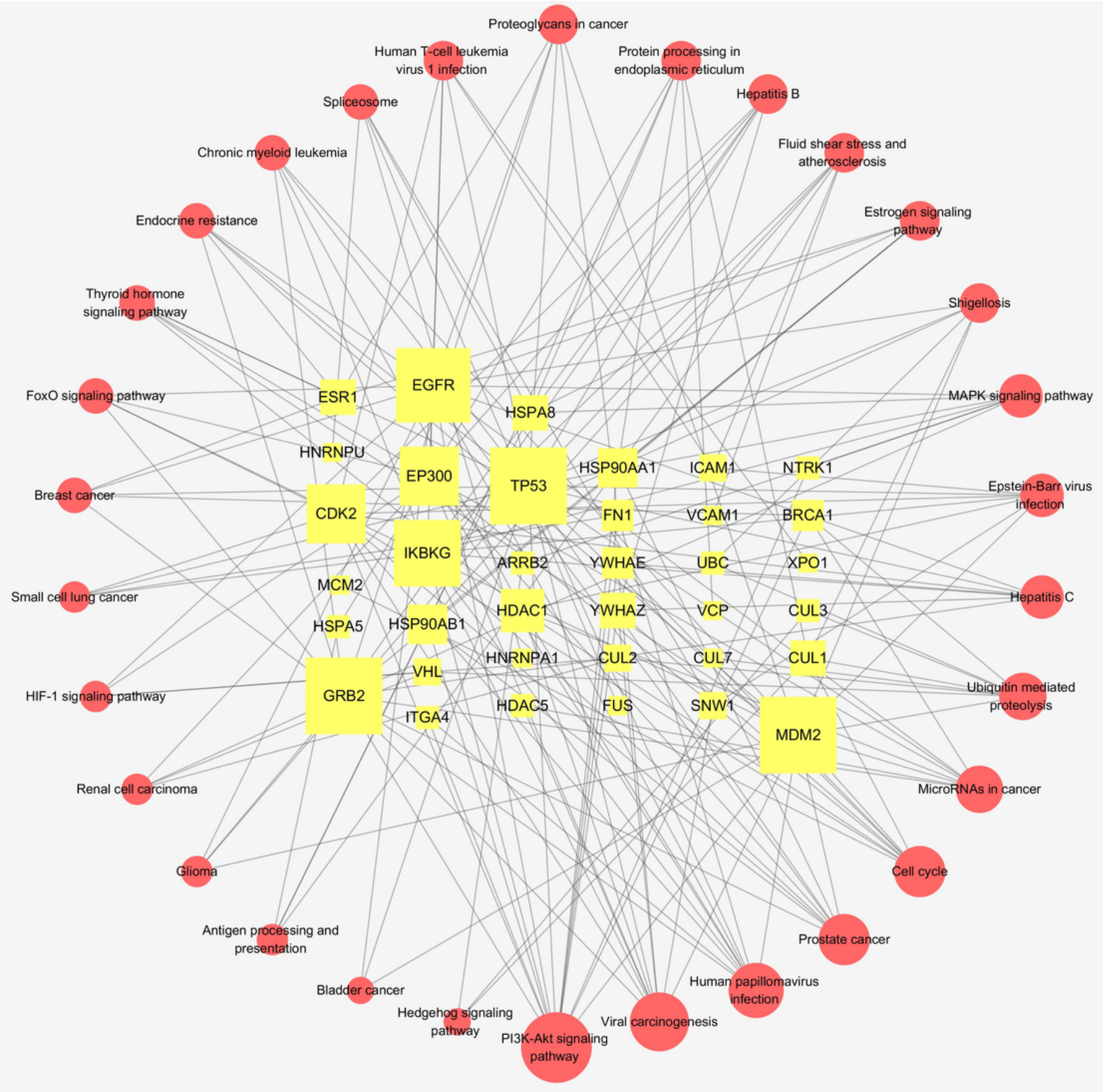

Figure 8

Target-pathway network. The round nodes in the center represent the important hub nodes, and the smaller round nodes in the middle ring represent the other nodes. The outermost triangles represent the related pathways. 


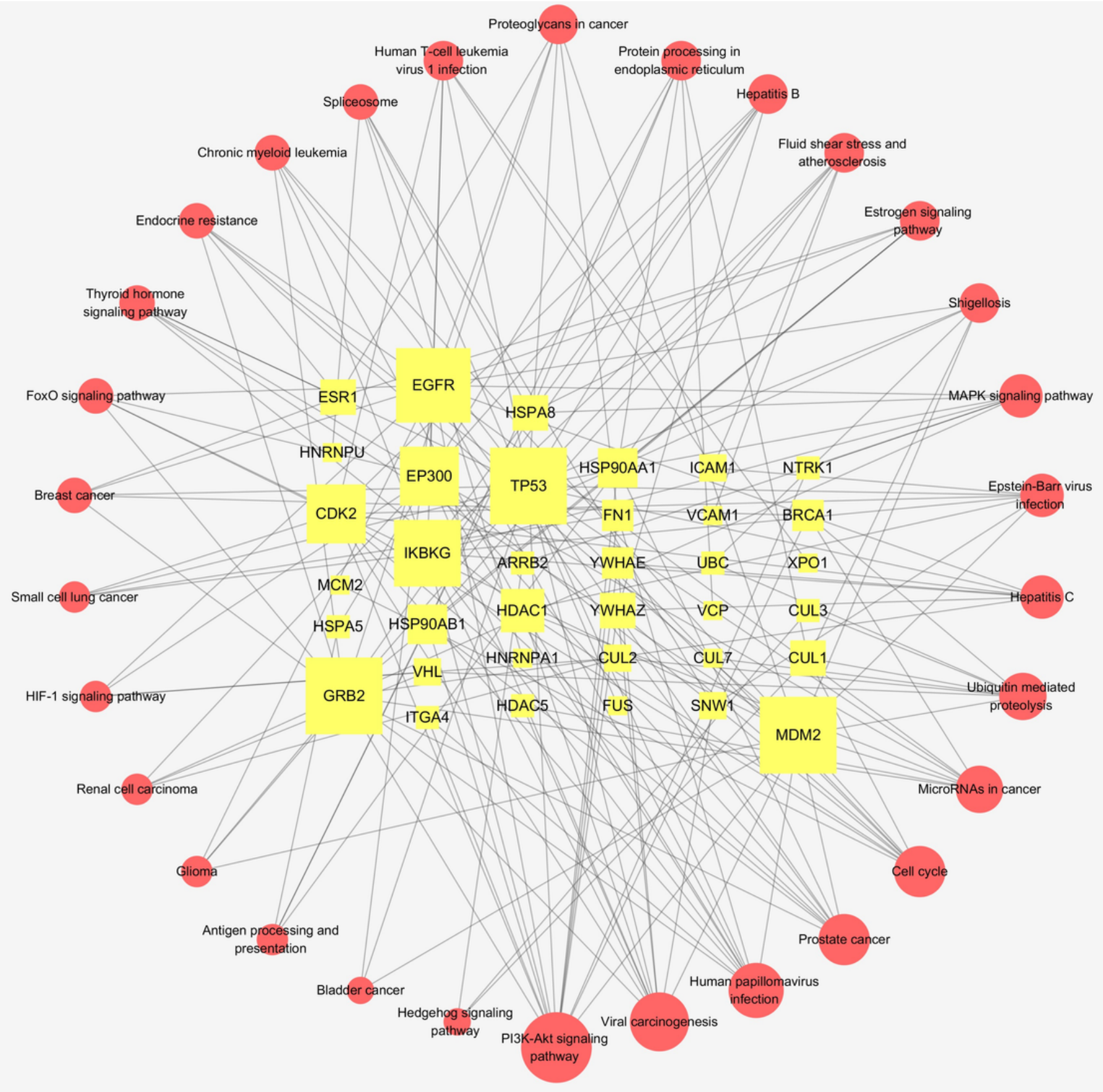

Figure 8

Target-pathway network. The round nodes in the center represent the important hub nodes, and the smaller round nodes in the middle ring represent the other nodes. The outermost triangles represent the related pathways. 


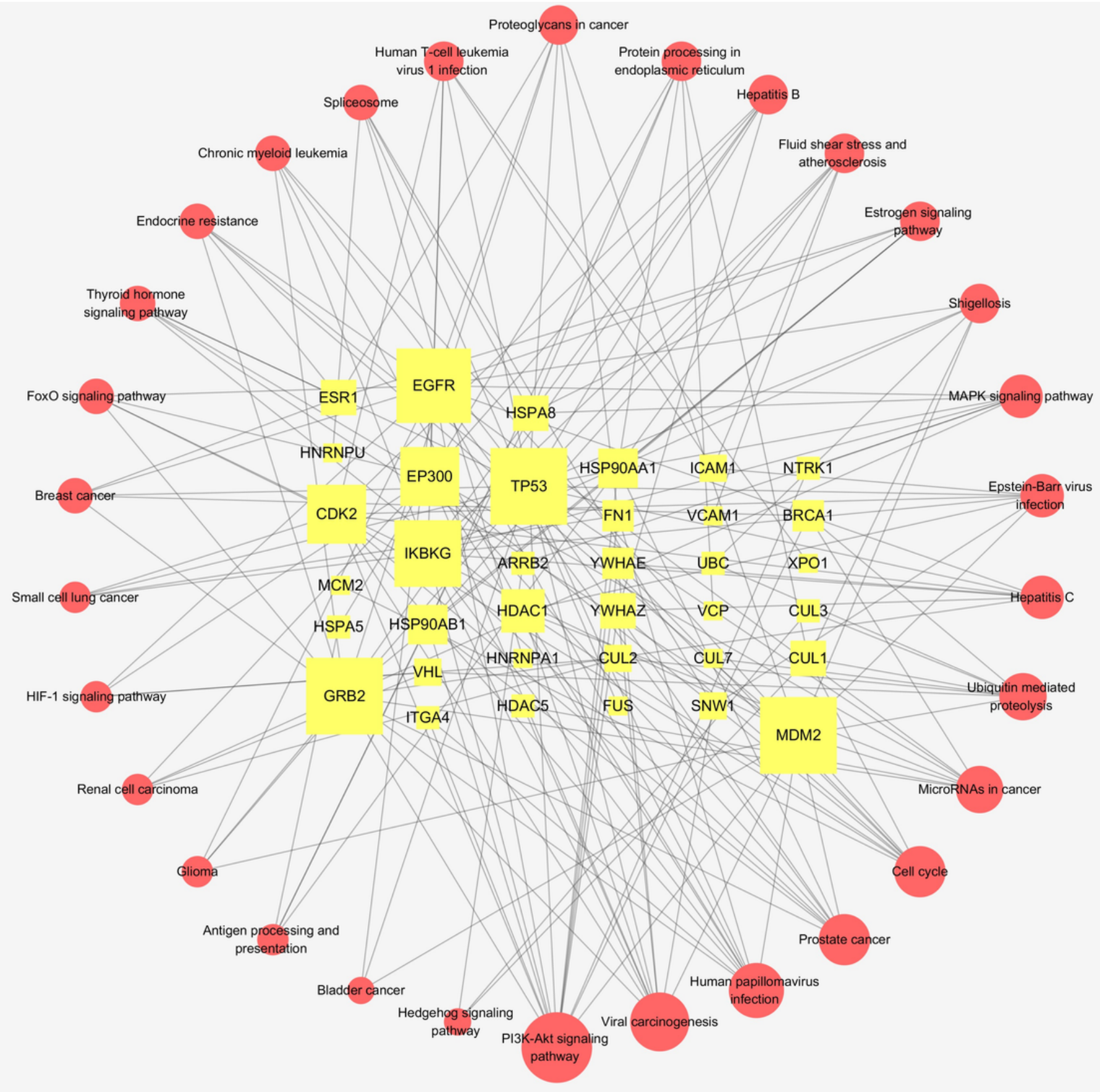

Figure 8

Target-pathway network. The round nodes in the center represent the important hub nodes, and the smaller round nodes in the middle ring represent the other nodes. The outermost triangles represent the related pathways. 

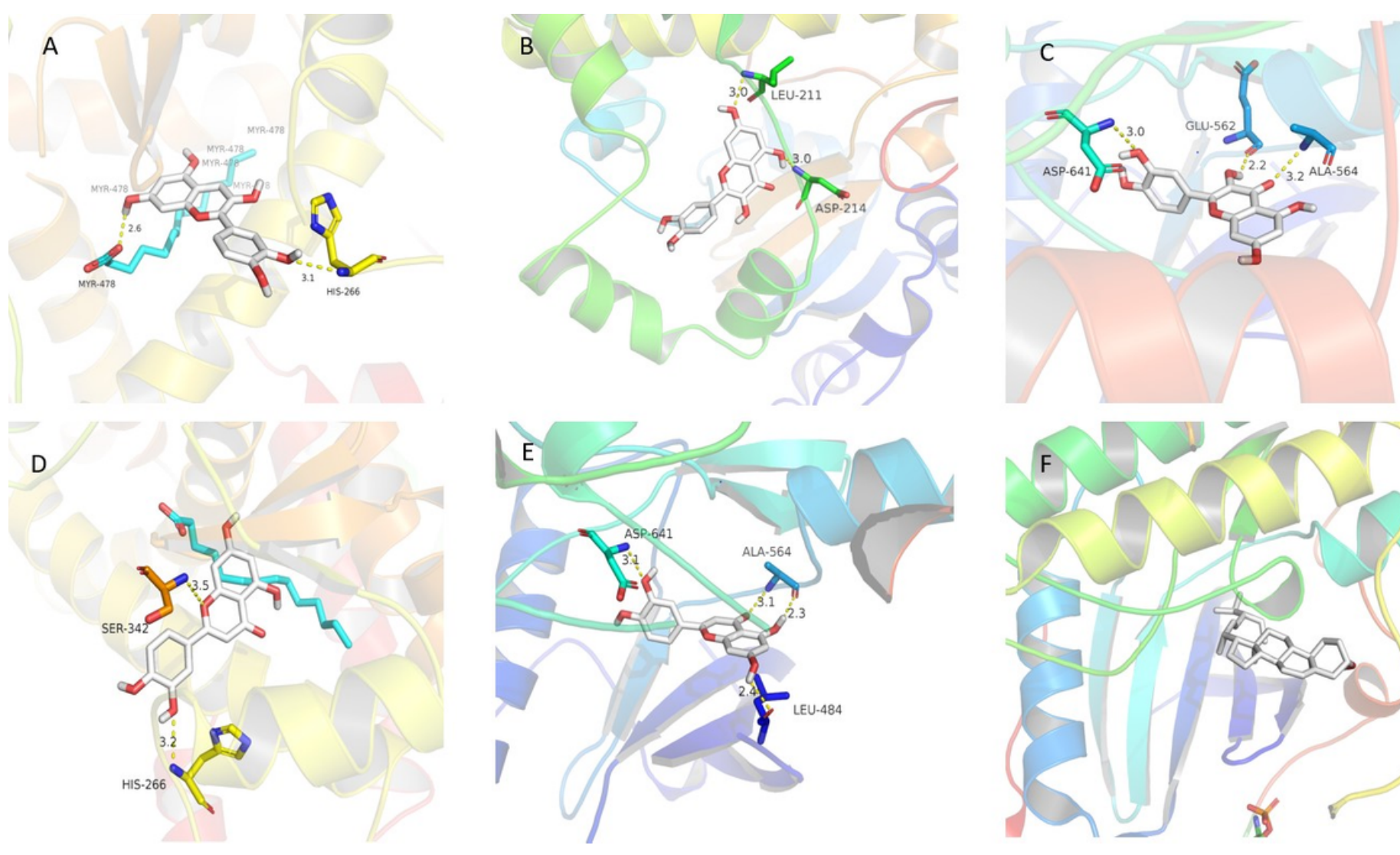

\section{Figure 9}
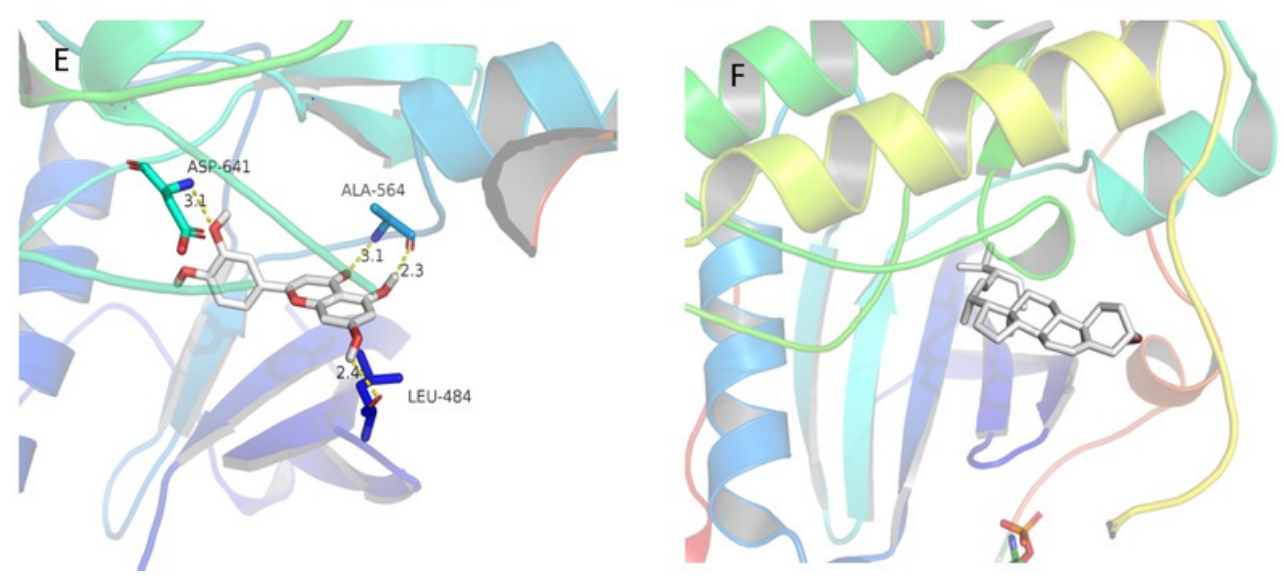

Six molecular docking diagram are shown. $\mathrm{A} \square \mathrm{B} \square \mathrm{C}$ correspond to the docked complexes of quercetin with PPARG, CYP3A4, VEGFA. D■E correspond to the docked complexes of luteolin with PPARG, VEGFA. F correspond to the docked complexes of Stigmasterol with IL-6. 

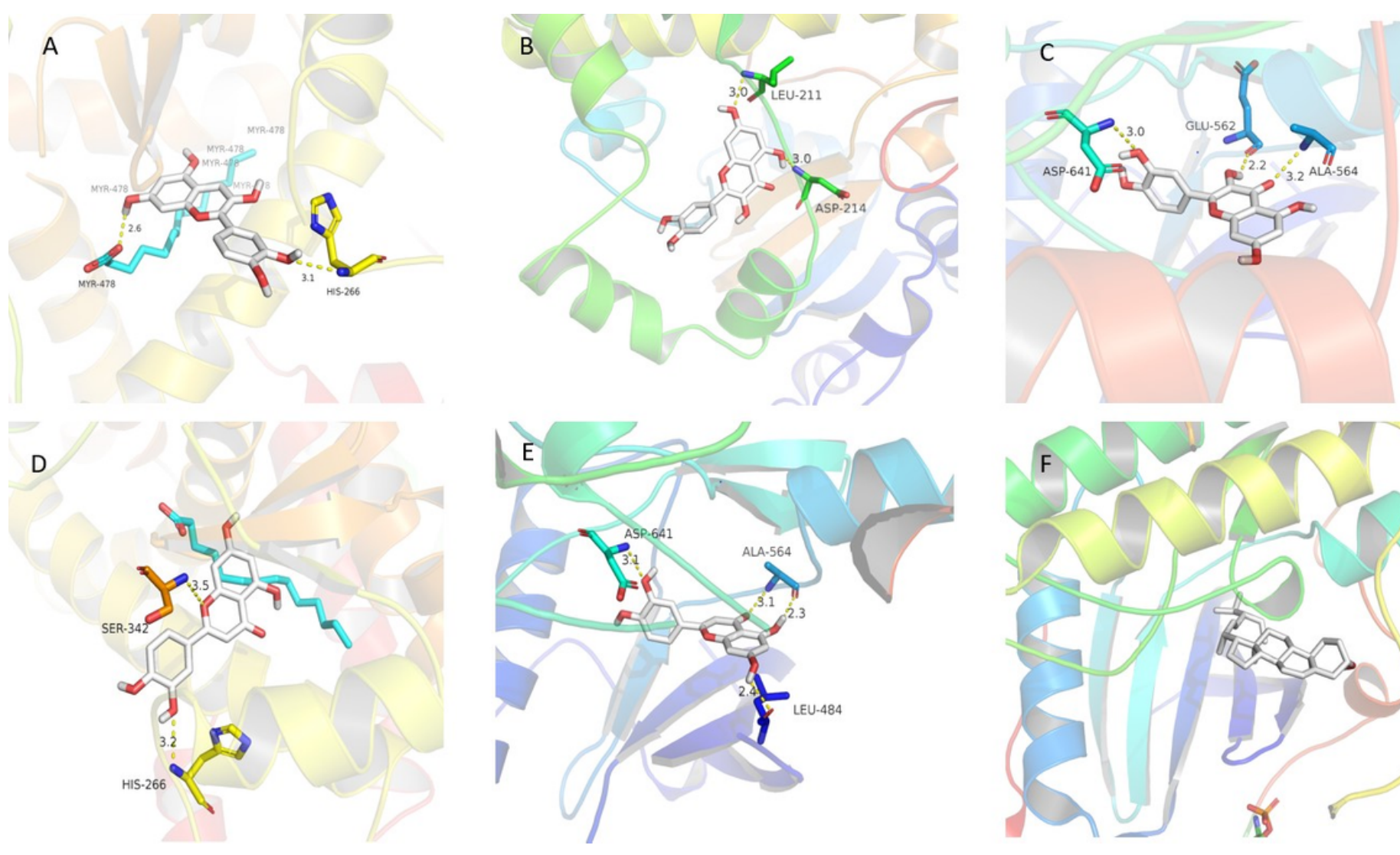

\section{Figure 9}
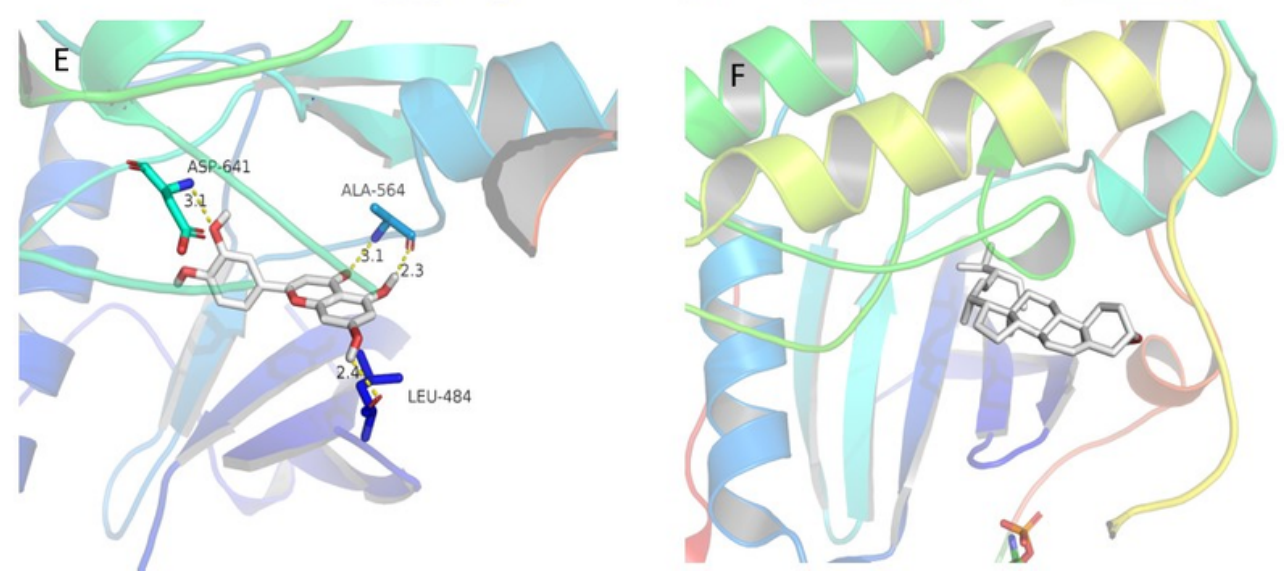

Six molecular docking diagram are shown. $\mathrm{A} \square \mathrm{B} \square \mathrm{C}$ correspond to the docked complexes of quercetin with PPARG, CYP3A4, VEGFA. D■E correspond to the docked complexes of luteolin with PPARG, VEGFA. F correspond to the docked complexes of Stigmasterol with IL-6. 

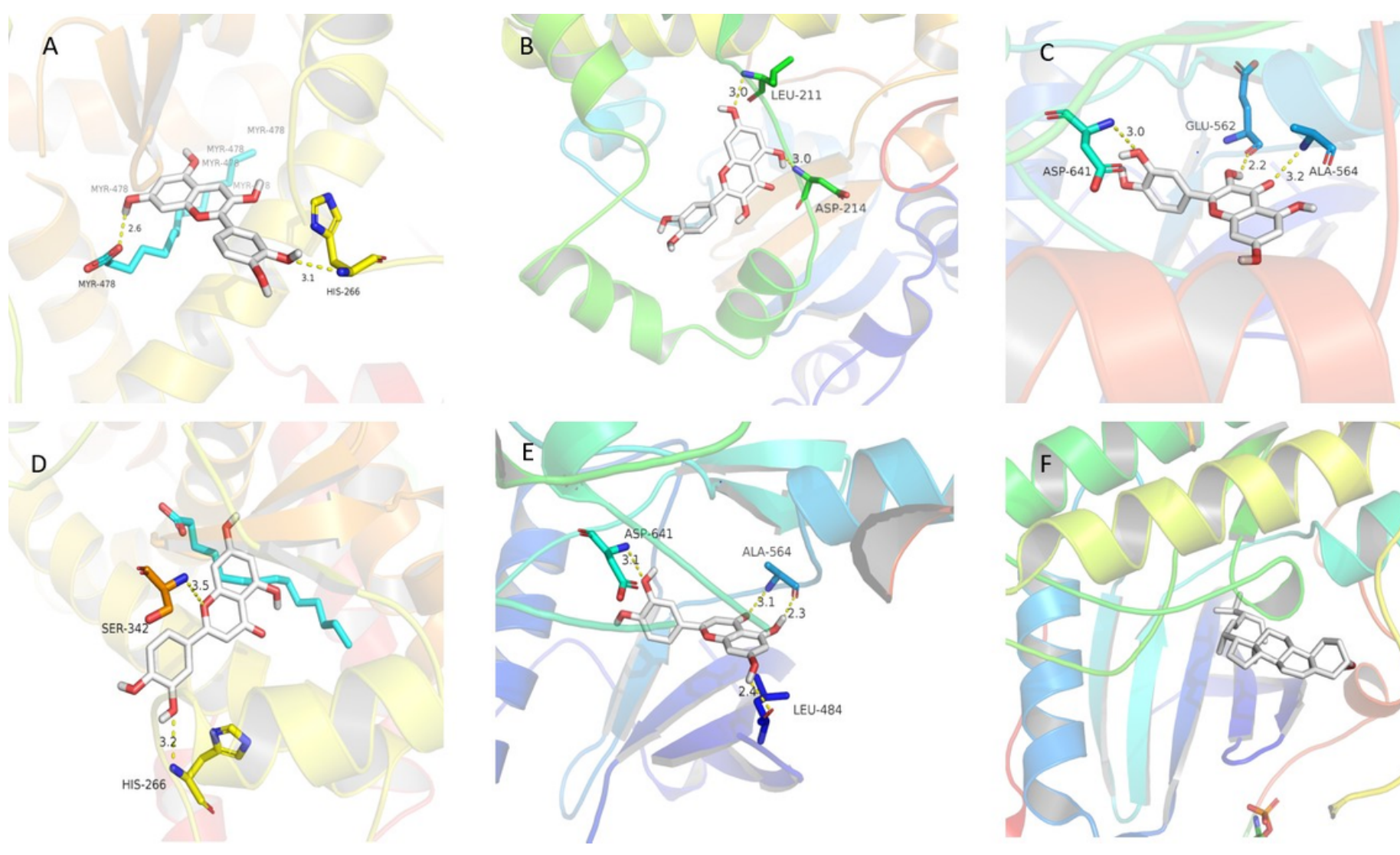

\section{Figure 9}
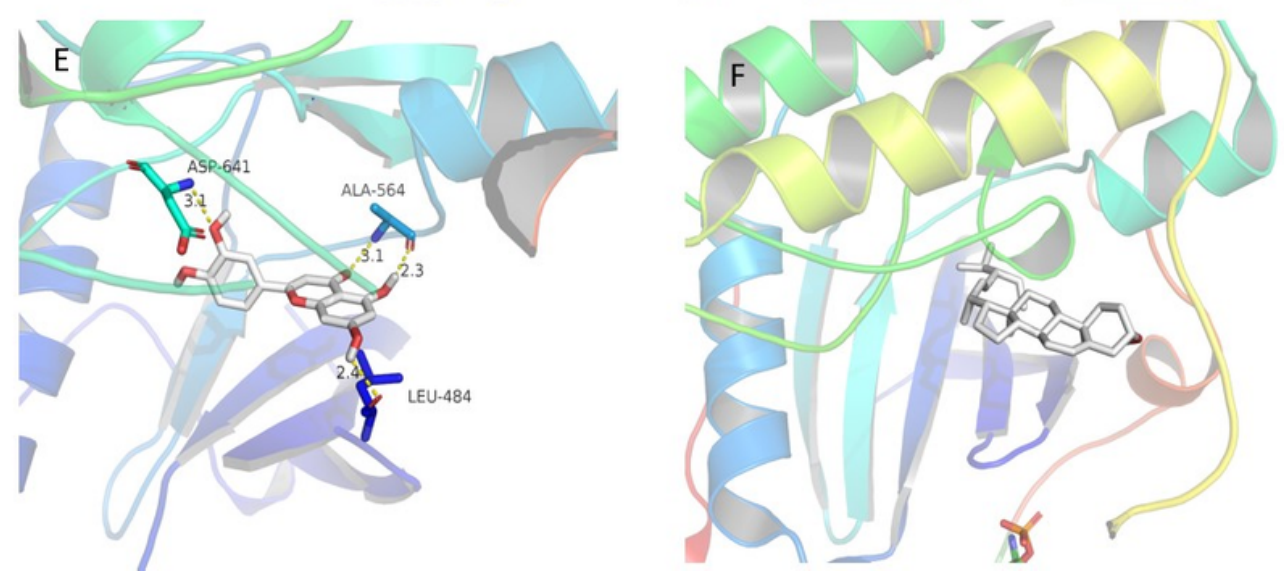

Six molecular docking diagram are shown. $\mathrm{A} \square \mathrm{B} \square \mathrm{C}$ correspond to the docked complexes of quercetin with PPARG, CYP3A4, VEGFA. D■E correspond to the docked complexes of luteolin with PPARG, VEGFA. F correspond to the docked complexes of Stigmasterol with IL-6. 